TRANSACTIONS OF THE

AMERICAN MATHEMATICAL SOCIETY

Volume 355, Number 7 , Pages $2733-2753$

S 0002-9947(03)03272-0

Article electronically published on March 19, 2003

\title{
A CLASSIFICATION AND EXAMPLES OF RANK ONE CHAIN DOMAINS
}

\author{
H. H. BRUNGS AND N. I. DUBROVIN
}

\begin{abstract}
A chain order of a skew field $D$ is a subring $R$ of $D$ so that $d \in D \backslash R$ implies $d^{-1} \in R$. Such a ring $R$ has rank one if $J(R)$, the Jacobson radical of $R$, is its only nonzero completely prime ideal. We show that a rank one chain order of $D$ is either invariant, in which case $R$ corresponds to a real-valued valuation of $D$, or $R$ is nearly simple, in which case $R, J(R)$ and (0) are the only ideals of $R$, or $R$ is exceptional in which case $R$ contains a prime ideal $Q$ that is not completely prime. We use the group $\mathcal{M}(R)$ of divisorial $R$-ideals of $D$ with the subgroup $\mathcal{H}(R)$ of principal $R$-ideals to characterize these cases. The exceptional case subdivides further into infinitely many cases depending on the index $k$ of $\mathcal{H}(R)$ in $\mathcal{M}(R)$. Using the covering group $\mathbb{G}$ of SL $(2, \mathbb{R})$ and the result that the group ring $T \mathbb{G}$ is embeddable into a skew field for $T$ a skew field, examples of rank one chain orders are constructed for each possible exceptional case.
\end{abstract}

\section{INTRODUCTION}

A subring $R$ of a skew field $D$ is called total if $d$ in $D$ and $d$ not in $R$ implies that the inverse $d^{-1}$ is contained in $R$. It follows that for such rings $R$ the lattice of right ideals as well as the lattice of left ideals is linearly ordered by inclusion; $R$ is a chain domain. Conversely, any chain domain $R$ is Ore and is a total subring of its skew field of quotients $D$. The total subrings of fields are exactly valuation rings, corresponding to valuation functions into linearly ordered groups. In particular, if we take nontrivial subgroups $G$ of the additive group $(\mathbb{R},+, \leq)$ of the reals as value groups, then we obtain the commutative valuation rings of rank one. Such a ring can also be characterized as a maximal subring of a field, or as a valuation ring with exactly one nonzero prime ideal. In the non-commutative case we must distinguish between prime ideals and completely prime ideals: An ideal $B \neq R$ of a ring $R$ is prime if $I_{1} I_{2} \subseteq B$ implies $I_{1} \subseteq B$ or $I_{2} \subseteq B$ for ideals $I_{1}$ and $I_{2}$ of $R$. If $a b \in B$ implies $a \in B$ or $b \in B$ for elements $a, b$ in $R$, then $B$ is called completely prime. A total subring $R$ of a skew field $D$ will be called a chain domain of rank one if $R$ has exactly one nonzero completely prime ideal. This ideal will then be $J(R)$, the Jacobson radical of $R$.

Received by the editors April 10, 2002 and, in revised form, October 9, 2002.

2000 Mathematics Subject Classification. Primary 16L30, 16K40, 16W60; Secondary 20F29, $20 \mathrm{~F} 60$.

Key words and phrases. Exceptional chain domains, skew field, valuation, cone, covering group. The first author was supported by NSERC.

The second author was supported by RFBR and DFG (grant no. 98-01-04110). 
We prove in Theorem 1.9 that a rank one chain domain $R$ is either invariant, i.e., all one-sided ideals are two-sided, or it is nearly simple in which case $R, J(R)$, and (0) are its only ideals, or $R$ is exceptional in which case $R$ contains a prime ideal that is not completely prime. The exceptional rank one chain domains are classified further with the help of the group $\mathcal{M}(R)$ of divisorial $R$-ideals and the subgroup $\mathcal{H}(R)$ of $\mathcal{M}(R)$ of principal $R$-ideals. The lattice of two-sided $R$-ideals is then determined by the index $k$ of $\mathcal{H}(R)$ in $\mathcal{M}(R)$, and we say that $R$ is exceptional of type $\left(C_{k}\right)$.

These results are proved in the more general case of cones $P$ in groups $G$ where a cone $P$ of $G$ is a subsemigroup of $G$ so that $g \in G \backslash P$ implies $g^{-1} \in P$.

That rank one chain domains are either invariant, nearly simple or exceptional was proved in [4. Invariant rank one chain orders of $D$ correspond to valuation functions from $D^{*}$ into $(\mathbb{R},+, \leq)$. Nearly simple chain domains were constructed in [8, 16, [5] and 3]. The construction of exceptional rank one chain domains, however, appeared to be elusive even though Posner in [19] hinted that such rings might exist, and the classification of hypercyclic rings by Osofsky in 18 is complete only if such rings do not exist. I. N. Herstein had considered the problem and this existence problem was also encountered in 14. We construct in this paper exceptional rank one chain domains of any type $\left(C_{k}\right)$ : Theorem 4.4 and Corollary 4.6. We do this by first constructing exceptional cones $P_{k}$ of type $\left(C_{k}\right)$ in subgroups $H_{k}$ of the universal covering group $\mathbb{G}$ of $\mathrm{SL}(2, \mathbb{R})$, Theorem 3.8 , and then apply Dubrovin's result in [11], where he constructs an exceptional rank one chain ring of type $\left(C_{1}\right)$ associated with a cone $\mathbb{P}$ in $\mathbb{G}$.

\section{Chain Domains and Cones}

1.1. Basic properties. A ring $R$ is a right chain ring, if the set of all right ideals of $R$ is linearly ordered with respect to inclusion. Left chain rings and chain rings are defined similarly. A chain domain $R$ has a classical skew field of quotients $D$ and can therefore be considered as a total subring of $D([7])$.

A subsemigroup $P$ of a group $G$ is called a cone of $G$ if $G=P \cup P^{-1}$ and $P$ is a pure cone if in addition $P \cap P^{-1}=\{e\}$. There is a close connection between cones $P$ in a group $G$ and right or left orders: if $P$ is a cone of $G$ and $a, b \in G$, then $\underset{\ell}{\leq}$ defined by $a \leq b$ if and only if $a^{-1} b \in P$ defines a left preorder, and $a \leq b$

if and only if $b a^{-1} \in P$ defines a right preorder on $G$. The relations " $\frac{>}{r}$ " and " $\leq$ " are right orders and left orders on $G$ respectively if and only if the cone $P$ is pure. Finally, if $P$ is pure, then the right order defined by $P$ agrees with the left order defined by $P$ if and only if $a P=P a$ for all $a$ in $G$, i.e., $P$ is invariant under all inner automorphisms of $G$. The group $G$ is then linearly ordered.

Let $P$ be a cone of a group $G$. A nonempty subset $I$ of $G$ is called a left $P$-ideal if $P I \subseteq I$ and $I \subseteq P a$ for a suitable element $a$ in $G$. The second condition is satisfied for any $I \neq G$ provided $I$ satisfies the first condition. If in addition $I \subseteq P$, we say $I$ is a left ideal. Right $P$-ideals, $P$-ideals and right ideals and ideals are defined similarly. An ideal $B$ of $P$ is called a prime ideal if $B \neq P$ and $a P b \subseteq B$ implies $a \in B$ or $b \in B$ for $a, b \in P$. If $a b \in B$ implies $a \in B$ or $b \in B$ for the ideal $B \neq P$ of $P$, then $B$ is called completely prime.

We collect elementary properties of a cone $P$ in $G$. We can assume that $P \neq G$. Let $U(P)=P \cap P^{-1}$, the subgroup of units of $P$. 
a): $J(P)=P \backslash U(P)$ is the maximal right and the maximal left ideal of $P$; it is the Jacobson radical of $P$ and it is a completely prime ideal of $P$.

b): The set of right (left) $P$-ideals in $G$ is linearly ordered with respect to inclusion. We define $I_{1} \leq I_{2}$ if and only if $I_{1} \supseteq I_{2}$ for right $P$-ideals $I_{1}$ and $I_{2}$.

To see this, one considers first principal right $P$-ideals $a P$ and $b P$ in $G$. Then either $a^{-1} b \in P$ and $b P \subseteq a P$ or $b^{-1} a \in P$ and $a P \subseteq b P$. If $I_{2} \nsubseteq I_{1}$, then there exists $a$ in $I_{2} \backslash I_{1}$ and $I_{1} \subset a P \subseteq I_{2}$ follows.

c): There is a one-to-one correspondence between the set of cones $P^{\prime} \neq G$ in $G$ that contain a cone $P$ and the set of completely prime ideals $B$ of $P$.

Proof. Let $P \subseteq P^{\prime} \subset G$ be cones in $G$. Then $j^{\prime} \in J\left(P^{\prime}\right)$ and $j^{\prime} \notin P$ implies $j^{\prime-1} \in P$, a contradiction. Hence, $J\left(P^{\prime}\right) \subseteq J(P)$ and $P^{\prime}=P \cup\left(P \backslash J\left(P^{\prime}\right)\right)^{-1}$. Conversely, if $B \subseteq J(P)$ is a completely prime ideal in $P$, then $P^{\prime}=P \cup(P \backslash B)^{-1}$ is a cone $\neq G$ in $G$.

d): Let $I$ be an ideal in $P$ with $I \neq P$ and $Q=\bigcap I^{n} \neq \emptyset$. Then $Q$ is a completely prime ideal.

Proof. If $c \in P \backslash Q$ and $c a \in Q$ for some $a$ in $P$, then there exists $n_{0}$ with $c \notin$ $I^{n_{0}}$. However, for any $n$ there exist $a_{i}, b_{j} \in I$ with $c a=a_{1} \ldots a_{n_{0}} b_{1} \ldots b_{n}$. Then $a_{1} \ldots a_{n_{0}}=c d$ for some $d$ in $P$ and $a=d b_{1} \ldots b_{n} \in I^{n}$ follows. Hence, $a \in Q$ and $Q$ is a completely prime ideal.

e): A $P$-ideal $I$ will be right principal and left principal if and only if $I=$ $z P=P z$ for some $z \in G$.

Proof. Let $I=z_{1} P=P z_{2}$ with $z_{1}, z_{2} \in G$. Then $z_{2}=z_{1} a, z_{1}=b z_{2}$ for some $a, b \in P$. Hence, $b z_{1} a=z_{1}$. Since $I$ is an ideal, there exists $b^{\prime}$ in $P$ with $b z_{1}=z_{1} b^{\prime}$ and $z_{1}=z_{1} b^{\prime} a$ follows. Therefore, $b^{\prime} a=1$ and $a \in U(P)$, and $P z_{2}=z_{1} P=z_{1} a P=$ $z_{2} P$.

f): Let $P$ be a cone in $G$. The set $\mathcal{H}(P)$ of all principal $P$-ideals of $G$ forms a group with ideal multiplication as the operation. $\mathcal{H}(P)$ is isomorphic to a subgroup of $(\mathbb{R},+, \leq)$ if $J(P)$ is the only completely prime ideal of $P$.

Proof. If $I_{1}=z_{1} P=P z_{1}$ and $I_{2}=z_{2} P=P z_{2}$, then $I_{1} I_{2}=z_{1} P z_{2} P=z_{1} z_{2} P$ and $\left(z_{1} P\right)^{-1}=z_{1}^{-1} P$. It follows that $\mathcal{H}(P)$ is a group with $P$ as identity. To prove the second statement let $P \supset z P=P z$. Then $\bigcap(z P)^{n}=\emptyset$ since otherwise $\bigcap(z P)^{n}$ is a completely prime ideal $\neq J(P)$ by d). $\mathcal{H}(P)$ is therefore an ordered Archimedean group and the statement follows from Hölder's Theorem (see [13]).

g): A right $P$-ideal $I$ is a principal right $P$-ideal if and only if $I J(P) \neq I$.

Proof. If $I=z P$, then $z P \supset I J(P)=z J(P)$. Conversely, if $I$ is not principal as a right $P$-ideal, then for $a \in I$ there exists $b \in I$ with $a P \subset b P, a=b j \in I J(P)$, $j \in J(P)$, and $I J(P)=I$.

We single out cones with the property in $\mathrm{f}$ ):

Definition 1.1. A cone $P$ of a group $G$ has rank one if $J(P)$ is the only completely prime ideal of $P$. 
It follows from the definitions that a subring $R$ of a skew field $D$ is total if and only if the semigroup $R^{*}=(R \backslash\{0\}, \cdot)$ is a cone in the group $D^{*}$.

This relationship between a cone in a group and a chain domain is generalized in the next definition.

Definition 1.2. A total subring $R$ in a skew field $D$ is said to be associated with a cone $P$ in a group $G$ if the following conditions hold:

i): $G$ is a subgroup of $D^{*}$, the multiplicative group of $D$.

ii): Every element $d$ in $D^{*}$ can be written as $d=g_{1} u_{1}=u_{2} g_{2}$ with $g_{1}, g_{2}$ in $G$ and $u_{1}, u_{2}$ in $U(R)$ so that $P g_{1} P=P g_{2} P$.

iii): $R \cap G=P$.

We also say in this case that the cone $P$ is associated with the chain domain $R$.

Proposition 1.3. Let the total subring $R$ of the skew field $D$ be associated with the cone $P$ of the group $G$. Then:

i): $I_{0} \rightarrow I_{0} R$ defines an isomorphism from the lattice of right $P$-ideals to the lattice of nonzero right $R$-ideals. The inverse of this mapping assigns $I \cap G$ to the nonzero right $R$-ideal $I$.

ii): The correspondence defined in i) preserves the properties of being an ideal, a completely prime ideal, a prime ideal, and a principal right ideal.

Proof. i) If $I_{0}$ is a right $P$-ideal, then two nonzero elements $a, b$ in $I_{0} R$ have the form $a=g_{1} u_{1}, b=g_{2} u_{2}$ for $g_{i} \in I_{0}$ and $u_{i} \in U(R)$. We can assume that $g_{1} P \subseteq g_{2} P$, and $g_{1}=g_{2} p, p \in P$ follows. Therefore, $a \pm b=g_{2}\left(p u_{1} \pm u_{2}\right) \in I_{0} R$; this shows that $I_{0} R$ is a right $R$-ideal, since $g I_{0} \subseteq P \subseteq R$ for some $g \in G \subseteq D$. Further, if $g \in I_{0} R \cap G$ for a right $P$-ideal $I_{0}$, then $g=h g^{\prime} u$ for $h \in I_{0}, g^{\prime} \in P$ and $u \in U(R)$. It follows that $h g^{\prime} \in I_{0}$ and $u \in U(R) \cap G=U(P)$; hence, $g \in I_{0}$ and $I_{0} R \cap G=I_{0}$. Similarly, one can show that $I \cap G$ is a right $P$-ideal if $I$ is a right $R$-ideal and that $(I \cap G) R=I$.

For ii) we only show that the right $P$-ideal $I_{0}$ is a $P$-ideal if and only if $I_{0} R$ is an $R$-ideal. Let $r \in R$ and $h \in I_{0}$, a $P$-ideal. Then $r=p_{1} u_{1}$ for $p_{1} \in P, u_{1} \in U(R)$ and $r h=p_{1} u_{1} h=p_{1} k u_{2}$ for $u_{1} h=k u_{2}$ with $u_{2} \in U(R)$ and $h, k \in G$. By ii) of Definition 1.2 we have $P h P=P k P ; k \in I_{0}$ follows and $r h \in I_{0} R$, which shows that $I_{0} R$ is also a left $R$-module and then an $R$-ideal. Conversely, if $I_{0} R$ is an $R$-ideal for a right $P$-ideal $I_{0}$, then $I_{0}=I_{0} R \cap G$ is a $P$-ideal.

Some variations of the results in this section can be found in [12] and [6].

1.2. Divisorial ideals. We consider certain $P$-ideals for a cone $P$ which will form a group in case $P$ has rank one.

Definition 1.4. Let $P$ be a cone in a group $G$. The divisorial closure $\widehat{I}$ of a right $P$-ideal $I$ is the intersection of all principal right $P$-ideals containing $I$ :

$$
\widehat{I}=\bigcap_{h P \supseteq I} h P .
$$

A right $P$-ideal $I$ is called divisorial if $\widehat{I}=I$.

If we replace the cone $P$ by a total subring $R$, we obtain the definition of the divisorial closure of a right $R$-ideal and of a divisorial right $R$-ideal. In addition, we assume that a divisorial right $R$-ideal is nonzero.

We collect a list of properties: 
Let $P$ be a cone in a group $G, I$ a $P$-right ideal. Then:

a): $\widehat{I} \supseteq I$

b): $\widehat{\widehat{I}}=\widehat{I}$

c): $\widehat{g I}=g \widehat{I}$ for any $g$ in $G$;

d): $I$ is non-divisorial if and only if $J(P)$ is not a principal right ideal and there exists an element $z$ in $G$ with $\widehat{I}=z P$ and $I=z J(P)$. If, in addition, $I$ is a $P$-ideal and $\operatorname{rank} P=1$, then $\widehat{I}=z P=P z$ and $I=z J(P)=J(P) z$.

The properties a, b, and c follow directly from the definition. To prove d) we will write $J$ instead of $J(P)$ and assume that $\widehat{I} \supset I$ and that $z \in \widehat{I} \backslash I$. Then $\widehat{I} \supseteq z P \supset I$ and $\widehat{I}=z P$ follows; then $I=z J$, since $z j P \supseteq I$ for some $j \in J(P)$ leads to a contradiction. This also shows that $J$ is not a principal right ideal. If $J$ is not a principal right ideal, then $c P \supseteq z J$ implies $z^{-1} c P \supseteq J$ and $z^{-1} c P \supseteq P, c P \supseteq z P$ for $c, z \in G$. This means that $\widehat{I}=z P$ for $I=z \bar{J}$ and hence $\widehat{I} \supset I$. If $z P$ is a $P$-ideal, then certainly $z J$ is a $P$-ideal. Conversely, if $z J$ is an ideal, then $z P$ is an ideal, since otherwise there is an $a \in P$ and a $j \in J$ with $a z j=z$, a contradiction. Finally, we assume that $\widehat{I} \neq I$ and $I$ is a $P$-ideal and that $P$ has rank one. Then $\widehat{I}=z P$ and the left order $O_{\ell}(I)=\{g \in G \mid g \widehat{I} \subseteq \widehat{I}\} \neq G$ contains the cone $P$ as well as the cone $z P z^{-1}$ both of which are maximal. It follows that $P=z P z^{-1}$, $P z=z P=\widehat{I}$ and $J z=z J=I$.

We list a property that was proved in the proof of $\mathrm{d}$ ):

e): $I$ is a $P$-ideal if and only if $\widehat{I}$ is a $P$-ideal.

The next result shows that in the correspondence between right $R$-ideals and right $P$-ideals, divisorial right ideals correspond to each other if the chain domain $R$ is associated with the cone $P$.

Proposition 1.5. Let $R$ be a total subring of the skew field $D$ associated with the cone $P$ in a group $G$. Then the right $P$-ideal $I$ is divisorial if and only if the right $R$-ideal $I R$ is divisorial.

Proof. Assume $I$ is divisorial, i.e., $I=\widehat{I}=\bigcap_{h P \supseteq I} h P$. Then $I R \subseteq h R$ for all $h \in G$ with $h P \supseteq I$, and $I R \subseteq \bigcap_{h P \supseteq I} h R$. To show the reverse inclusion, let $d \in \bigcap h R$ for $h P \supseteq I$ and $d=h r_{h}=g m$ for $g \in G, m \in U(R)$. Hence, $g=h r_{h} m^{-1} \in$ $h R \cap G=h P$ and $g \in \bigcap h P=I, d \in I R$ follows. Now assume that $A$ is a divisorial right $R$-ideal, $A=\bigcap_{d R \supset A} d R$. Any such $d=g m$ for $g \in G, m \in U(R)$. Hence, $A \cap G=(\bigcap d R) \cap G=\bigcap(g R \cap G)=\bigcap g P$, which shows that $A \cap G$ is divisorial and $A \cap G$ is nonempty, since $A$ is nonzero.

For any subset $I$ of a group $G$ we define the following three subsets of $G$ : the right order $O_{r}(I)=\{g \in G \mid I g \subseteq I\}$, the left order $O_{\ell}(I)=\{g \in G \mid g I \subseteq I\}$, and the inverse $I^{-1}=\{g \in G \mid I g I \subseteq I\}$.

It follows that $I^{-1}=\left\{g \in G \mid g I \subseteq O_{r}(I)\right\}=\left\{g \in G \mid I g \subseteq O_{\ell}(I)\right\}$.

We have the following two properties where $P$ is a cone in the group $G$ :

f): If $I$ is a right $P$-ideal, then $O_{\ell}(I)$ is a cone of $G$ and $O_{r}(I)$ is an over cone of $P$. Further, $I$ is a right $O_{r}(I)$-ideal and a left $O_{\ell}(I)$-ideal, and $I^{-1}$ is a right $O_{\ell}(I)$-ideal and a left $O_{r}(I)$-ideal. 
For a proof we observe that for any $g$ in $G$ either $g I \subseteq I$ and $g \in O_{\ell}(I)$ or $I \subset g I$ and $g^{-1} \in O_{\ell}(I)$. The rest of the statements follow immediately.

g): $O_{r}(J(P))=O_{\ell}(J(P))=P$, and $J(P)^{2} \neq J(P)$ implies that $J(P)=$ $z P=P z$ for some $z \in P$.

The first statement follows from Property c) in Section 1.1 since $O_{r}(J(P)) \supset$ $P$ implies that $j^{-1} J(P) \subseteq J(P)$ for some $j \in J(P)$. Hence, $J(P) \subseteq j J(P)$, a contradiction that shows $O_{r}(J(P))=P$ and similarly $O_{\ell}(J(P))=P$.

The second statement follows from Property g) in Section 1.1, its left symmetric version, and Property e) in Section 1.1.

Even though one can consider the groupoid of all divisorial $P$-ideals for a cone $P$ of arbitrary rank (see also [2]), we restrict ourselves to the rank one case:

Definition 1.6. Let $P$ be a cone of rank one. Then $\mathcal{M}(P)$ is the set of all divisorial $P$-ideals together with the operation "*" defined by:

$$
I_{1} * I_{2}=\widehat{I_{1} I_{2}} \text { for } P \text {-ideals } I_{1}, I_{2} \text {. }
$$

We have the following result:

Theorem 1.7. Let $P$ be a cone of rank one in a group $G$. Then:

a) $\mathcal{M}(P)$ is a linearly ordered group;

B) The inverse of an element I in $\mathcal{M}(P)$ is $I^{-1}$;

r) $\mathcal{H}(P)$ is a subgroup of $\mathcal{M}(P)$.

Proof. We show first that the operation defined in Definition 1.6 is associative.

On the set of all $P$-ideals we define a relation $I_{1} \sim I_{2}$ if and only if $\widehat{I}_{1}=\widehat{I}_{2}$; this is an equivalence relation.

We are going to show next that for $P$-ideals $I_{1}, I_{2}$ the following equivalence holds:

$$
I_{1} I_{2} \sim \widehat{I}_{1} \widehat{I}_{2} .
$$

If $I_{1}=\widehat{I}_{1}$ and $I_{2}=\widehat{I}_{2}$, then $(+)$ is trivially true. If $I_{1} \neq \widehat{I}_{1}$, then $\widehat{I}_{1}=z P \supset$ $z J(P)=I_{1}$ and $J=J(P)$ is not right principal. Also $\widehat{I}_{1}=z P=P z$ is a $P$-ideal by Property d).

The equivalence $(+)$ holds therefore if and only if the following equivalence holds:

$$
J I_{2} \sim P \widehat{I}_{2}=\widehat{I}_{2}
$$

Hence, if $J I_{2}=I_{2}$, we are done. Otherwise, $J I_{2} \subset I_{2}$ and $I_{2}=P d$ follows for some $d$ in $G$ by the left symmetric version of Property g) in Section 1.1. Since $I_{2}$ is an ideal, we have $d P \subseteq P d, P \subseteq d^{-1} P d$ and the equality $d^{-1} P d=P$ since $P$ has rank one. Then $d P=P d=I_{2}, d J=J d$ and $J I_{2}=J d=d J \sim d P=I_{2}$ which proves the equivalence $(++)$ and hence also $(+)$ in this case.

Finally, we must prove $(+)$ if $I_{1}=\widehat{I}_{1}$ and $\widehat{I}_{2} \supset I_{2}$. Then, as above, $\widehat{I}_{2}=a P=$ $P a \supset a J=J a=I_{2}$ for some $a$ in $G$. The equivalence (+) then holds if and only if the equivalence $I_{1} J \sim \widehat{I}_{1} P=I_{1}$ holds. Using the right symmetric version of arguments used in the proof of $(++)$, one shows that $I_{1} J \sim I_{1}$. This proves $(+)$.

If $I_{1} \sim I_{1}^{\prime}$ and $I_{2} \sim I_{2}^{\prime}$ for $P$-ideals $I_{1}, I_{1}^{\prime}, I_{2}, I_{2}^{\prime}$, then $I_{1} I_{2} \sim \widehat{I_{1}} \widehat{I_{2}}=\widehat{I_{1}^{\prime}} \widehat{I_{2}^{\prime}} \sim I_{1}^{\prime} I_{2}^{\prime}$. Hence $\mathcal{M}(P)$ is a factor monoid of the monoid of all $P$-ideals, and the operation * given in the definition for $\mathcal{M}(P)$ is associative.

Next we show that $\widehat{I I^{-1}}=P$ for $I$ a $P$-ideal, and $\widehat{I^{-1} I}=P$ follows from similar arguments. Since $I$ is a $P$-ideal, $I^{-1}$ is a $P$-ideal. 
If $I I^{-1}=P$, we are done; otherwise $I I^{-1} \subseteq J(P)=J$. If $I I^{-1} \subseteq P z \subseteq J$ for some $z \in J$, then $I I^{-1} z^{-1} \subseteq P$ and $I^{-1} z^{-1} \in \bar{I}^{-1}$ which implies $z^{-1} \in O_{r}\left(I^{-1}\right)=$ $P$, since $P$ has rank one. This is a contradiction since $z \in J$, and $I I^{-1}=J, J \neq P z$ for all $z \in P$ remains as the only possibility to be considered. It then follows from Property g) that $J^{2}=J, J$ is not a principal right ideal, and hence $\widehat{I I^{-1}}=\widehat{J}=P$.

In order to complete the proof of $\alpha$ ) and $\beta$ ) we show that $I^{-1}$ is a divisorial $P$-ideal for $I$ a $P$-ideal. If on the contrary, $I^{-1}=z J \subset z P=\widehat{I^{-1}}$ and $J$ is not a principal right ideal, then $z J I \subseteq P$ by the definition of $I^{-1}$, and by ( + ) it follows that $\widehat{z J} \widehat{I} \subseteq \widehat{P}=P$. Since $\widehat{z J}=z P$, we obtain $z I \subseteq z \widehat{I} \subseteq P$, and hence $z \in I^{-1}=z J$, a contradiction.

This shows that $\mathcal{M}(P)$ is a group and that $\beta$ ) holds. For $I_{1} \supseteq I_{2}$ in $\mathcal{M}(P)$ we define $I_{1} \leq I_{2}$ and $\mathcal{M}(P)$ then is a linearly ordered group with $P$ as identity. Elements in $\mathcal{H}(P)$ have the form $I=z P=P z$ for some $z$ in $G$ with $\widehat{z P}=z P$ and $(z P)^{-1}=z^{-1} P=P z^{-1}$; see $\left.\mathrm{f}\right)$ in Section 1.1 and $\left.\gamma\right)$ follows. This proves the theorem.

Corollary 1.8. Let $P$ be a cone of rank one in a group $G$. Then $\mathcal{M}(P)$ and $\mathcal{H}(P)$ are Archimedean groups.

Proof. Let $B \subset P$ be a divisorial ideal. If $B \subset J(P)=J$ or $B=J \neq J^{2}$, then $\bigcap B^{n}=\emptyset$ by Property d) in Section 1.1. If $J=J^{2}$, we have $\widehat{J}=P$ and hence $\bigcap B^{n}=\emptyset$ in all cases, and $B^{n+1} \subset B^{n}$. Then $\widehat{B}^{n+1} \subseteq B^{n}$, since there are no further right ideals between $B^{n+1}$ and $\widehat{B}^{n+1}$. This implies $\bigcap \widehat{B}^{n}=\emptyset$, and it follows that $\mathcal{M}(P)$ and $\mathcal{H}(P)$ are Archimedean; see also Property f) in Section 1.1.

Related results can be found in [12] and [2].

1.3. The classification of rank one cones. The groups $\mathcal{M}(P)$ and $\mathcal{H}(P)$ will be used to classify rank one cones $P$ in groups $G$ based on the lattice of their ideals. In the following theorem and proof we will write $J$ instead of $J(P)$.

Theorem 1.9. Let $P$ be a cone of rank one in a group $G$. Then exactly one of the following possibilities occurs:

A) : The cone $P$ is Archimedean, i.e., $a P=P a$ for all $a$ in $P$. We distinguish two possibilities in this case:

$\left.\mathbf{A}_{\mathbf{1}}\right): \mathcal{M}(P)=\mathcal{H}(P) \cong(\mathbb{Z},+, \leq)$, which is exactly the case when $J^{2} \neq J$. Then every $P$-ideal is a power of $J$ and the cone is called discrete.

$\left.\mathbf{A}_{\mathbf{2}}\right): \mathcal{M}(P) \cong(\mathbb{R},+, \leq)$ and $\mathcal{H}(P)$ is a dense subgroup of $\mathcal{M}(P)$.

$\mathbf{B})$ : The cone $P$ is nearly simple; i.e., $J$ is the only proper ideal in $P$. In this case $\mathcal{M}(P)=\mathcal{H}(P)=\{P\}$.

$\mathbf{C})$ : The cone $P$ is exceptional; i.e., there exists a prime ideal $Q$ in $P$ that is not completely prime. Then:

i): There are no further ideals between $J$ and $Q$.

ii): The ideal $Q$ is divisorial and $\mathcal{M}(P)=\operatorname{gr}\{Q\}$ is an infinite cyclic group. iii): $\bigcap Q^{n}=\emptyset$.

iv): There exists an integer $k \geq 0$ such that $\mathcal{H}(P)=\operatorname{gr}\left\{\widehat{Q^{k}}\right\}$. The cone $P$ is said to be of type $\left(C_{k}\right)$ in this case.

If $P$ is of type $\left(C_{0}\right)$, then

$$
\cdots \supset\left(Q^{n}\right)^{-1} \supset \cdots \supset Q^{-1} \supset P \supset J \supset Q \supset Q^{2} \supset Q^{3} \supset \cdots
$$


is the chain of $P$-ideals.

If $P$ is of type $\left(C_{1}\right)$, then

$$
\begin{aligned}
\cdots \supset Q^{-2} & =z^{-2} P \supset z^{-2} J \supset Q^{-1}=z^{-1} P \supset z^{-1} J \supset P \supset J \supset z P \\
& =Q \supset z J \supset z^{2} P=Q^{2} \supset z^{2} J \supset \cdots
\end{aligned}
$$

is the chain of $P$-ideals.

If $P$ is of type $\left(C_{k}\right), k \geq 2$, then

$$
\begin{aligned}
& \cdots \supset\left(Q^{k+1}\right)^{-1} \supset z^{-1} P \supset z^{-1} J \supset\left(Q^{k-1}\right)^{-1} \supset \cdots \\
& \supset Q^{-1} \supset P \supset J \supset Q \supset Q^{2} \supset \cdots \supset Q^{k-1} \supset z P \supset z J \\
& =Q^{k} \supset Q^{k+1} \supset \cdots \supset Q^{2 k-1} \supset z^{2} P \supset z^{2} J \\
& =Q^{2 k} \supset Q^{2 k+1} \supset \cdots
\end{aligned}
$$

is the chain of all $P$-ideals.

Proof. If $J$ is the only proper ideal of $P$, then $P$ is of type $B$.

Otherwise, let $Q=\bigcup I$ be the union of ideals of $P$ properly contained in $J$. If $J^{2}=J$ and $J \supset Q$, then $P$ is exceptional: for ideals $I_{1} \supset Q$ and $I_{2} \supset Q$ in $P$ we have $I_{1} \cdot I_{2} \supseteq J^{2}=J \supset Q$ and $Q$ is a prime ideal of $P$, not completely prime and no further ideal exists between $J$ and $Q$. The divisorial closure $\widehat{Q}$ of $Q$ is an ideal that cannot be equal to $J$, since $J$ would then be right principal. Hence, $\widehat{Q}=Q$ is the smallest positive element in the linearly ordered Archimedean group $\mathcal{M}(P)$, and $\mathcal{M}(P)=\operatorname{gr}\{Q\}$ is an infinite cyclic group. The subgroup $\mathcal{H}(P)$ has therefore the form $\mathcal{H}(P)=\operatorname{gr}\left\{\widehat{Q^{k}}\right\}$ for some $k \geq 0$; we say that $P$ is of type $\left(C_{k}\right)$.

We can now describe the $P$-ideals in each case $\left(C_{k}\right)$ if we recall (see Property d) in Section 1.2) that an ideal $I$ is either divisorial or of the form $c J=J c$ with $\widehat{I}=c P=P c$, some $c \in G$ and $J=J^{2}$. It will also follow from the rest of the proof that if $P$ is exceptional, then $J=J^{2}$ and $J \supset Q=\bigcup I$, where the ideals $I$ are properly contained in $J$, the prime ideal that is not completely prime.

In the case $\left(C_{0}\right)$ there are no principal ideals $\neq P$ and the group $\mathcal{M}(P)=\operatorname{gr}\{Q\}$ contains all $P$-ideals $\neq J$. In the case $\left(C_{1}\right)$ the ideal $Q=z P=P z$ is principal and $\mathcal{M}(P)=\mathcal{H}(P)$. In the case $\left(C_{k}\right), k \geq 2$, the ideal $\widehat{Q^{k}}$ is principal. However, $Q^{k}$ itself cannot be principal, since otherwise $Q^{k}=z P$ implies $Q^{k} J \neq Q^{k}$; hence, $Q J \neq Q$ and $Q$ is principal (see Property g) in Section 1.1). Hence $\widehat{Q^{k}}=z P=$ $P z \supset Q^{k}=z J=J z$ for an element $z$ in $P$.

It remains to consider the case where either $J \neq J^{2}$, or $J=J^{2}$ and $J=Q=\bigcup I$ for ideals $I$ properly contained in $J$. In this case we will prove that $a P=P a$ for all $a$ in $P$. If for some $a$ in $P$ the right ideal $a P$ is not a left ideal, then an element $c$ exists in $P$ with $c a P \supset a P$ and $c a j=a$ follows for an element $j$ in $J$. By assumption there exists an ideal $I \subseteq J$ with $j \in I$ and $\bigcap I^{n}=\emptyset$; we obtain the contradiction $a=c a j=c^{n} a j^{n} \in \bigcap I^{n}$. We have $P a \subseteq a P, P \subseteq a P a^{-1}$ and $P=a P a^{-1}$ since $P$ is of rank one. Therefore, $P a=a P$ for all $a$ in $P$ and $P$ is invariant.

If $J \neq J^{2}$, then $J=a P=P a$, for some $a$ in $P$, is the smallest positive element in the Archimedean group $\mathcal{M}(P)$. Hence, $\mathcal{M}(P)=\mathcal{H}(P)=\operatorname{gr}\{J\}$ is the group of all $P$-ideals.

If $J=J^{2}$ and $J=Q$, then $\mathcal{H}(P)$ is isomorphic to a dense subgroup of $(\mathbb{R},+, \leq)$ and $\mathcal{M}(P)$ is isomorphic to $(\mathbb{R},+\leq)$.

If $R$ is a chain order of rank one in a skew field $D$, then $R^{*}=R \backslash\{0\}$ is a cone in the group $D^{*}$. We say that $R$ has type $(A),\left(A_{1}\right),\left(A_{2}\right),(B),(C)$, or $\left(C_{k}\right)$ if and only if the cone $R^{*}$ is of the same type. 
The next result follows from Propositions 1.3 and 1.5 and Theorem 1.9.

Corollary 1.10. Let $P$ be a cone associated with the rank one chain domain $R$. Then $P$ and $R$ have the same type.

\section{The Universal Covering Group $\mathbb{G}$ of $\operatorname{SL}(2, \mathbb{R})$}

2.1. The group $\mathrm{SL}(2, \mathbb{R})$. By $\mathrm{SL}(2, \mathbb{R})$ we denote, as usual, the group of $2 \times 2$ matrices with real entries and determinant equal to 1 . Then

$$
\mathbb{U}=\left\{u=\left(\begin{array}{cc}
a & b \\
0 & a^{-1}
\end{array}\right) \mid a, b \in \mathbb{R}, a>0\right\}
$$

and

$$
\mathbb{S}=\left\{r(t)=\left(\begin{array}{cc}
\cos t & -\sin t \\
\sin t & \cos t
\end{array}\right) \mid t \in \mathbb{R}\right\}
$$

are two particular subgroups of $\mathbb{G}$. Every element $s \in \mathrm{SL}(2, \mathbb{R})$ can be written in a unique way as

$$
s=r(t) u \quad \text { for } \quad r(t) \in \mathbb{S} \text { with } 0 \leq t<2 \pi \quad \text { and } \quad u \in \mathbb{U} .
$$

To prove this claim, let $\left\{e_{1}, e_{2}\right\}$ be the standard basis of $\mathbb{R}^{2}$, the Euclidean plane, and let the elements of $\mathrm{SL}(2, \mathbb{R})$ be the representations of linear transformations of $\mathbb{R}^{2}$ with respect to the basis $\left\{e_{1}, e_{2}\right\}$. For every nonzero vector $\mathbf{a} \in \mathbb{R}^{2}$ there exists a unique element $t \in[0,2 \pi)$ with $\mathbf{a} /\|\mathbf{a}\|=e_{1} \cos t+e_{2} \sin t$; we write $\arg \mathbf{a}=t$ in this case.

Let $t=\arg s\left(e_{1}\right)$ for the given element $s \in \mathrm{SL}(2, \mathbb{R})$ and $r(-t) s=u \in \mathbb{U}$ for some element $u$, since $r(-t) s\left(e_{1}\right)=a e_{1}$ for $a>0$. Hence, $s=r(t) u$ and this representation is unique, since $\mathbb{U} \cap \mathbb{S}=\{I\}, I=\left(\begin{array}{ll}1 & 0 \\ 0 & 1\end{array}\right)$, the identity of $\operatorname{SL}(2, \mathbb{R})$.

2.2. The group $\mathbb{G}$. We are going to construct the universal covering group $\mathbb{G}$ of the group $\mathrm{SL}(2, \mathbb{R})$ in this section. We do this first for the subgroup $\mathbb{S}$ by fixing a symbol, say $x$, and by rewriting the additive group of the real numbers in multiplicative form:

$$
R=\left\{x^{t} \mid t \in \mathbb{R}\right\} ; \quad x^{t_{1}} \cdot x^{t_{2}}=x^{t_{1}+t_{2}} ; \quad x^{t_{1}} \leq x^{t_{2}} \Leftrightarrow t_{1} \leq t_{2} .
$$

Then $R$ is a linearly ordered group isomorphic to $(\mathbb{R},+, \leq)$. The mapping $\tau$ from $R$ to $\mathbb{S}$ with $\tau\left(x^{t}\right)=r(t)$ is a group epimorphism with the cyclic subgroup $\operatorname{gr}\left\{x^{2 \pi}\right\}$ as its kernel; $\tau$ is a cover of the Lie group $\mathbb{S}$. Next we define the covering group $\mathbb{G}$ of $\operatorname{SL}(2, \mathbb{R})$ as the set $\mathbb{G}=\left\{x^{t} u \mid x^{t} \in R, u \in \mathbb{U}\right\}$, the Cartesian product $R \times \mathbb{U}$, together with the following operation: If $x^{t_{1}} u_{1}, x^{t_{2}} u_{2}$ are two elements in $\mathbb{G}$ and $t_{2}=2 \pi k+\varphi$ for $k \in \mathbb{Z}$ and $\varphi \in[0,2 \pi)$, then $u_{1} r(\varphi) u_{2}=r(\psi) u$ in $\operatorname{SL}(2, \mathbb{R})$ for $u \in \mathbb{U}, \psi \in[0,2 \pi)$, and the product in $\mathbb{G}$ is defined as $x^{t_{1}} u_{1} \cdot x^{t_{2}} u_{2}=x^{t_{1}+2 \pi k+\psi} u$.

The mapping $\tau$ from above can be extended to a mapping from $\mathbb{G}$ to $\operatorname{SL}(2, \mathbb{R})$ by defining

$$
\tau\left(x^{t} u\right)=r(t) u .
$$

We want to prove that $\mathbb{G}$ is a group and that $\tau$ is an epimorphism from $\mathbb{G}$ onto $\mathrm{SL}(2, \mathbb{R})$.

Lemma 2.1. The mapping $\tau$ is onto $\mathrm{SL}(2, \mathbb{R})$, and if $a \cdot b=c$ for elements $a, b, c$ in $\mathbb{G}$, then $\tau(c)=\tau(a) \tau(b)$. 
Proof. The element $x^{t} u$ in $\mathbb{G}$ satisfies $\tau\left(x^{t} u\right)=r(t) u$ for the arbitrary element $r(t) u$ in $\operatorname{SL}(2, \mathbb{R}) ; \tau$ is onto. If $a=x^{t_{1}} u_{1}, b=x^{t_{2}} u_{2}$ in $\mathbb{G}, t_{2}=2 \pi k+\varphi, k \in \mathbb{Z}, \varphi \in[0,2 \pi)$ and if $u_{1} r(\varphi) u_{2}=r(\psi) u, \psi \in[0,2 \pi), u_{i}, u \in \mathbb{U}$, then $c=x^{t_{1}+2 \pi k+\psi} u$ and

$$
\begin{aligned}
\tau(c) & =r\left(t_{1}+2 \pi k+\psi\right) u=r\left(t_{1}\right) r(\psi) u=r\left(t_{1}\right) u_{1} r(\varphi) u_{2} \\
& =\tau(a) r(2 \pi k+\varphi) u_{2}=\tau(a) \tau(b),
\end{aligned}
$$

which proves the lemma.

Several special cases of the associative law for the operation defined for $\mathbb{G}$ are proved in the next few steps. We can consider $R$ as well as $\mathbb{U}$ as subgroups of $\mathbb{G}$ and the equations

$$
x^{t} \cdot u=x^{t} u, \quad x^{t_{1}} \cdot x^{t} u=x^{t_{1}+t} u, \quad \text { and } \quad x^{t} u \cdot u^{\prime}=x^{t} u u^{\prime}
$$

follow. We conclude also that $x^{t} \cdot a=x^{t} \cdot b$ implies $a=b$ for elements $a, b \in \mathbb{G}$.

Lemma 2.2. For any element $g=x^{t} u \in \mathbb{G}$ and any $m \in \mathbb{Z}$ the product $g \cdot x^{\pi m}$ is equal to $x^{t+\pi m} u$.

Proof. We have $\pi m=2 \pi k+\varphi$ with $k \in \mathbb{Z}$, and $\varphi=0$ if $m$ is even, and $\varphi=\pi$ if $m$ is odd. In both cases $u r(\varphi)=r(\varphi) u$ follows, which proves the statement of the lemma.

Lemma 2.3. For any $a, b \in \mathbb{G}$ and any integer $m \in \mathbb{Z}$ the following equalities hold:

$$
x^{\pi m} \cdot(a \cdot b)=\left(x^{\pi m} \cdot a\right) \cdot b=a \cdot\left(x^{\pi m} \cdot b\right) .
$$

Proof. Because of $(+)$ the first equation follows, and we can assume that $a=u \in \mathbb{U}$ and $b=x^{t} \in R$ in the second equation.

It remains to prove the following equality:

$$
\left(x^{\pi m} \cdot u\right) \cdot x^{t}=u \cdot\left(x^{\pi m} \cdot x^{t}\right)
$$

where $t=2 \pi k+\varphi, k \in \mathbb{Z}, \varphi \in[0,2 \pi)$ and $\operatorname{ur}(\varphi)=r(\psi) u^{\prime}$ for $\psi \in[0,2 \pi), u^{\prime} \in \mathbb{U}$.

Then $\left(x^{\pi m} \cdot u\right) \cdot x^{t}=x^{\pi m+2 \pi k+\psi} u^{\prime}$. We distinguish three cases in order to compute the right-hand side of the above equation.

In the first case, $m=2 k^{\prime}$ is even and the equality follows immediately.

In the second case, $\pi m=2 \pi k^{\prime}+\pi$ for some $k^{\prime} \in \mathbb{Z}$ and $\varphi<\pi$. Then

$$
u \cdot x^{2 \pi\left(k+k^{\prime}\right)+\varphi+\pi}=x^{2 \pi\left(k+k^{\prime}\right)} u \cdot x^{\pi+\varphi}=x^{2 \pi\left(k+k^{\prime}\right)+\pi+\psi} u^{\prime}=x^{\pi m+2 \pi k+\psi} u^{\prime}
$$

since $u r(\pi+\varphi)=u r(\pi) r(\varphi)=r(\pi) r(\psi) u^{\prime}=r(\pi+\psi) u^{\prime}$ in $\operatorname{SL}(2, \mathbb{R})$; the equation is proved in this case.

In the final case, $\pi m=2 \pi k^{\prime}+\pi$ for $k^{\prime} \in \mathbb{Z}$ and $\varphi \geq \pi$. The right-hand side of the above equation is then equal to

$$
u \cdot x^{2 \pi\left(k+k^{\prime}+1\right)+\varphi-\pi}=x^{2 \pi\left(k+k^{\prime}+1\right)-\pi+\psi} u^{\prime}=x^{\pi m+2 \pi k+\psi} u^{\prime},
$$

which proves the lemma.

Lemma 2.4. Let $u \in \mathbb{U}$ and $t \in(\pi m, \pi(m+1))$ for some $m \in \mathbb{Z}$. Then $u \cdot x^{t}=x^{t^{\prime}} u^{\prime}$ for $u^{\prime} \in \mathbb{U}$ and $t^{\prime} \in(\pi m, \pi(m+1))$.

Proof. Let $t=2 \pi k+\varphi$ for $k \in \mathbb{Z}, \varphi \in(0,2 \pi)$. If $m=2 k$ is even, then $\varphi \in$ $(0, \pi)$; hence $\sin \varphi>0$. It follows that for any $u=\left(\begin{array}{cc}a & b \\ 0 & a^{-1}\end{array}\right) \in \mathbb{U}$; the argument $\psi$ of $u r(\varphi)\left(e_{1}\right)=r(\psi) u^{\prime}\left(e_{1}\right)$ is also in $(0, \pi)$ since $\psi=\arg \left[\left(\begin{array}{ll}a & b \\ 0 & a^{-1}\end{array}\right)\left(\begin{array}{c}\cos \varphi \\ \sin \varphi\end{array}\right)\right]$ and $a^{-1} \sin \varphi>0$. Hence $t^{\prime}=2 \pi k+\psi \in(\pi m, \pi(m+1))$ as stated in the lemma. 
If $m=1+2 k$ is odd, then $t=2 \pi k+\varphi$ and $\varphi \in(\pi, 2 \pi)$. Then $\sin \varphi<0$ and $\sin \psi$ with $\operatorname{ur}(\varphi)=r(\psi) u^{\prime}$ is also negative with the above argument; hence, $\psi \in(\pi, 2 \pi)$ and $t^{\prime}=2 \pi k+\psi \in(\pi m, \pi(m+1))$.

Theorem 2.5. a): $\mathbb{G}$ is a group;

b): The mapping $\tau$ is a homomorphism from $\mathbb{G}$ onto $\mathrm{SL}(2, \mathbb{R})$;

c): The center of $\mathbb{G}$ is the infinite cyclic group generated by $x^{\pi}$.

Proof. To show that the operation defined for $\mathbb{G}$ is associative we consider three elements $x^{t_{i}} u_{i} \in G, i=1,2,3$ with $t_{i} \in \mathbb{R}$ and $u_{i} \in \mathbb{U}$ and the equation

$$
g_{1}=\left(x^{t_{1}} u_{1} \cdot x^{t_{2}} u_{2}\right) \cdot x^{t_{3}} u_{3}=x^{t_{1}} u_{1} \cdot\left(x^{t_{2}} u_{2} \cdot x^{t_{3}} u_{3}\right)=g_{2} .
$$

By Lemmas 2.2 and 2.3 this equation holds if and only if the following equation is true:

$$
\left(x^{t_{1}+\pi k} u_{1} \cdot x^{t_{2}+\pi m} u_{2}\right) \cdot x^{t_{3}+\pi n} u_{3}=x^{t_{1}+\pi k} u_{1} \cdot\left(x^{t_{2}+\pi m} u_{2} \cdot x^{t_{3}+\pi n} u_{3}\right)
$$

for integers $k, m$ and $n$.

It follows that it is sufficient to prove $(*)$ only in the case where $t_{1}, t_{2}, t_{3} \in[0, \pi)$.

For $g_{1}=x^{t} u^{\prime}$ and $g_{2}=x^{t^{\prime}} u^{\prime \prime}$ with $t, t^{\prime} \in \mathbb{R}, u^{\prime}, u^{\prime \prime} \in \mathbb{U}$ we apply Lemma 2.1 and obtain

$$
r(t) u^{\prime}=\left(r\left(t_{1}\right) u_{1} \cdot r\left(t_{2}\right) u_{2}\right) \cdot r\left(t_{3}\right) u_{3}
$$

and

$$
r\left(t^{\prime}\right) u^{\prime \prime}=r\left(t_{1}\right) u_{1} \cdot\left(r\left(t_{2}\right) u_{2} \cdot r\left(t_{3}\right) u_{3}\right)
$$

in $\operatorname{SL}(2, \mathbb{R})$ where the operation is associative, and therefore $r(t) u^{\prime}=r\left(t^{\prime}\right) u^{\prime \prime}$ follows. This implies $u^{\prime}=u^{\prime \prime}$ and $t-t^{\prime}=2 \pi k$ for some $k \in \mathbb{Z}$. It remains to show that $k=0$.

We apply Lemma 2.4 and obtain $u_{1} x^{t_{2}}=x^{t_{2}^{\prime}} u_{1}^{\prime}$ for $u_{1}^{\prime} \in \mathbb{U}, t_{2}^{\prime} \in[0, \pi) ; u_{1}^{\prime} u_{2}$. $x^{t_{3}}=x^{\tilde{t}_{3}} \widetilde{u}$ for $\widetilde{u} \in \mathbb{U}, \widetilde{t}_{3} \in[0, \pi) ; u_{2} x^{t_{3}}=x^{t_{3}^{\prime}} u_{2}^{\prime}$ for $u_{2}^{\prime} \in \mathbb{U}, t_{3}^{\prime} \in[0, \pi)$; and $u_{1} x^{t_{2}+t_{3}^{\prime}}=x^{t_{2,3}} u_{1}^{\prime \prime}$ for $u_{1}^{\prime \prime} \in \mathbb{U}$ and $t_{2,3} \in[0, \pi)$.

Therefore:

$$
\begin{aligned}
g_{1}=\left(x^{t_{1}} u_{1} \cdot x^{t_{2}} u_{2}\right) \cdot x^{t_{3}} u_{3} & =\left(x^{t_{1}+t_{2}^{\prime}} u_{1}^{\prime} u_{2}\right) \cdot x^{t_{3}} u_{3} \\
& =x^{t_{1}+t_{2}^{\prime}+\widetilde{t_{3}}} \widetilde{u} u_{3},
\end{aligned}
$$

and

$$
\begin{aligned}
g_{2}=x^{t_{1}} u_{1} \cdot\left(x^{t_{2}} u_{2} \cdot x^{t_{3}} u_{3}\right) & =x^{t_{1}} u_{1} \cdot x^{t_{2}+t_{3}^{\prime}} u_{2}^{\prime} u_{3} \\
& =x^{t_{1}+t_{2,3}} u_{1}^{\prime \prime} u_{2}^{\prime} u_{3} .
\end{aligned}
$$

Hence, $t=t_{1}+t_{2}^{\prime}+\widetilde{t}_{3}$ and $t^{\prime}=t_{1}+t_{2,3}$ and therefore

$$
t-t^{\prime}=t_{2}^{\prime}+\widetilde{t}_{3}-t_{2,3}=2 \pi k \text {. }
$$

However, $t_{2}^{\prime}+\widetilde{t}_{3}$ and $t_{2,3}$ both belong to $[0,2 \pi)$ and $k=0$ and the associative law follows for the operation defined for $\mathbb{G}$.

Since $\mathbb{G}$ has $e=x^{0} E$, for $E=\left(\begin{array}{ll}1 & 0 \\ 0 & 1\end{array}\right)$, as the identity and $x^{t} u$ has $u^{-1} x^{-t}$ as its inverse, $\mathbb{G}$ is indeed a group; this proves a).

The statement b) was proved in Lemma 2.1. It follows from Lemma 2.3 that $\operatorname{gr}\left\{x^{\pi}\right\}$ is contained in the center $Z(\mathbb{G})$ of $\mathbb{G}$. Conversely, if $x^{t} u \in Z(\mathbb{G})$ for $t \in \mathbb{R}$ and $u \in \mathbb{U}$, then an application of Lemma 2.1 shows that $r(t) u$ is in $Z(\operatorname{SL}(2, \mathbb{R}))$. 
Hence $r(t) u= \pm r(0), u=\left(\begin{array}{ll}1 & 0 \\ 0 & 1\end{array}\right)$, and $t=\pi k$ for some $k \in \mathbb{Z}$ follows. Therefore $x^{t} u \in \operatorname{gr}\left\{x^{\pi}\right\}$, which proves c) and the theorem.

See also [1] for the fact that $\mathbb{G}$ is right orderable, but not locally indicable.

2.3. The representation of the group $\mathbb{G}$. To each element $g=x^{t} u \in \mathbb{G}$ we can assign the projection $v(g)=v\left(x^{t} u\right)=x^{t} \in R$. The mapping $V: \mathbb{G} \rightarrow \operatorname{Aut}(R, \leq)$ is defined as $V_{g}\left(x^{t}\right)=v\left(g x^{t}\right)$ for $g \in \mathbb{G}, x^{t} \in R$. That $V_{g}$ is indeed an automorphism of $(R, \leq)$ follows from the next result.

Lemma 2.6. For $g \in \mathbb{G}$ let $V_{g}$ be defined as above. Then:

a): $V_{g_{1} g_{2}}=V_{g_{1}} \circ V_{g_{2}}$ for $g_{1}, g_{2} \in \mathbb{G}$.

b): $V_{g}$ is the identity mapping if and only if $g$ is the identity element in $\mathbb{G}$.

c): The stabilizer $\operatorname{st}\left(x^{t}\right)=\left\{g \in \mathbb{G} \mid V_{g}\left(x^{t}\right)=x^{t}\right\}$ is equal to $x^{t} \mathbb{U} x^{-t} \cong \mathbb{U}$, which is an Ore group.

d): $V_{g}$ is an automorphism of $(R, \leq)$ for every $g \in \mathbb{G}$.

Proof. To prove a) we compute $v\left(g_{1} g_{2} x^{t}\right)$ and $v\left(g_{1} v\left(g_{2} x^{t}\right)\right)$. Let $g_{1}=x^{t_{1}} u_{1}, g_{2}=$ $x^{t_{2}} u_{2}$ for $u_{i} \in \mathbb{U}$. Then $g_{1} g_{2} x^{t}=x^{t_{1}} u_{1} x^{t_{2}} u_{2} x^{t}=x^{t_{1}} u_{1} x^{t_{2}} x^{t^{\prime}} u^{\prime}$ for some $u^{\prime} \in$ $\mathbb{U}, t^{\prime} \in \mathbb{R}$ with $u_{2} x^{t}=x^{t^{\prime}} u^{\prime}$. Further, $x^{t_{1}} u_{1} x^{t_{2}+t^{\prime}} u^{\prime}=x^{t_{1}+\widetilde{t}} \widetilde{u} u^{\prime}$ for $u_{1} x^{t_{2}+t^{\prime}}=x^{\tilde{t}} \widetilde{u}$ for $\widetilde{u} \in \mathbb{U}, \widetilde{t} \in \mathbb{R}$. It follows that $v\left(g_{1} g_{2} x^{t}\right)=x^{t_{1}+\widetilde{t}}$ and that $v\left(g_{1} v\left(g_{2} x^{t}\right)\right)=$ $v\left(x^{t_{1}} u_{1} x^{t_{2}+t^{\prime}}\right)=x^{t_{1}+\tilde{t}}$; this proves a).

To prove b), assume $g=x^{t_{1}} u$ and $V_{g}\left(x^{t}\right)=x^{t}$ for all $t \in \mathbb{R}$. For $t=0$ it follows that $t_{1}=0$. We consider $t=\frac{\pi}{2}$ and assume that $u=\left(\begin{array}{cc}a & b \\ 0 & a^{-1}\end{array}\right)$. Then $V_{u}\left(x^{\frac{\pi}{2}}\right)=x^{\frac{\pi}{2}}$ implies that

$$
\arg \left[\left(\begin{array}{cc}
a & b \\
0 & a^{-1}
\end{array}\right)\left(\begin{array}{cc}
0 & -1 \\
1 & 0
\end{array}\right)\left(\begin{array}{l}
1 \\
0
\end{array}\right)\right]=\arg \left(\begin{array}{c}
b \\
a^{-1}
\end{array}\right)=\frac{\pi}{2} .
$$

Hence, $b=0$ and $u=\left(\begin{array}{cc}a & 0 \\ 0 & a^{-1}\end{array}\right)$. Finally, for $t=\frac{\pi}{4}$ we must have

$$
\arg \left[\left(\begin{array}{cc}
a & 0 \\
0 & a^{-1}
\end{array}\right)\left(\begin{array}{c}
\frac{1}{\sqrt{2}} \\
\frac{1}{\sqrt{2}}
\end{array}\right)\right]=\frac{\pi}{4}
$$

and $a=a^{-1}=1$ follows; hence, $g=e$, the identity in $\mathbb{G}$, and b) follows.

To prove c) we observe that $\operatorname{st}\left(x^{0}\right)=\left\{x^{t_{1}} u_{1} \in \mathbb{G} \mid V_{g}\left(x^{0}\right)=x^{t_{1}}=x^{0}\right\}$ equals $\mathbb{U}$. Hence, $V_{g}\left(x^{t}\right)=x^{t} \mathbb{U} x^{-t} \cong \mathbb{U}$. These stabilizers are Ore groups in the sense that the group ring $T \mathbb{U}$ over a skew field $T$ is an Ore domain. This is true since $\mathbb{U}$ is the semidirect product of the following two torsion free abelian groups:

$$
A=\left\{\left(\begin{array}{cc}
a & 0 \\
0 & a^{-1}
\end{array}\right) \mid 0<a \in \mathbb{R}\right\} \quad \text { and } B=\left\{\left(\begin{array}{ll}
1 & b \\
0 & 1
\end{array}\right) \mid b \in \mathbb{R}\right\} .
$$

This proves $\mathrm{c}$ ).

Finally, we want to prove d). Since $V_{x^{t}}$ is an automorphism of $(R, \leq)$, it follows from a) that it is enough to show that $V_{u}$ is an automorphism of $(R, \leq)$ for any $u \in \mathbb{U}$. We show first that $x^{t_{2}}>x^{t_{1}}$ implies $V_{u}\left(x^{t_{2}}\right)>V_{u}\left(x^{t_{1}}\right)$ which then implies that $V_{u}$ is one-to-one and order-preserving. By Lemma 2.4 and Theorem 2.5(c) we can assume $t_{1}, t_{2} \in[0, \pi)$. It then follows that $t_{2}-t_{1} \in(-\pi, \pi)$, and in addition $t_{2}-t_{1}>0$ if and only if

$$
\sin \left(t_{2}-t_{1}\right)=\operatorname{Det}\left(\begin{array}{ccc}
\cos t_{1} & \cos t_{2} \\
\sin t_{1} & \sin t_{2}
\end{array}\right)>0 .
$$

We have $u r\left(t_{i}\right)=r\left(d_{i}\right) u_{i}$ for $d_{i} \in[0, \pi)$ and $d_{i}=\arg \left(u\left(\begin{array}{c}\cos t_{i} \\ \sin t_{i}\end{array}\right)\right) \in[0, \pi)$. Then $\operatorname{Det}\left(u\left(\begin{array}{c}\cos t_{i} \cos t_{2} \\ \sin t_{1} \sin t_{2}\end{array}\right)\right)>0$, since $\operatorname{Det}(u)>0$, and, as in the previous argument, 
$d_{2}>d_{1}$ follows. This shows that $x^{d_{2}}=V_{u}\left(x^{t_{2}}\right)>x^{d_{1}}=V_{u}\left(x^{t_{1}}\right)$ for $t_{2}>t_{1}$ and that $V_{u}$ is order-preserving and one-to-one.

It remains to show that $V_{u}$ is onto, and by Lemma 2.4 and Theorem 2.5 (c) it is enough to show that $V_{u}$ maps the interval $\left[x^{0}, x^{\pi}\right]$ onto the interval $\left[x^{0}, x^{\pi}\right]$. This, however, follows from the fact that $V_{u}\left(x^{0}\right)=x^{0}, V_{u}\left(x^{\pi}\right)=x^{\pi}$ and that $V_{u}$ is continuous.

We will prove next a technical result which will be used several times.

Lemma 2.7. Let $g=x^{t} u \in \mathbb{G}$ with $t=\pi k+t_{0}$ and $x^{t_{1}} \in R$ with $t_{1}=\pi m+t_{10}$ for $k, m \in \mathbb{Z}$ and $t_{0}, t_{10} \in[0, \pi)$. Assume that $\left(\begin{array}{c}a \\ b\end{array}\right) \in \mathbb{R}^{2}$ with $\arg \left(\begin{array}{c}a \\ b\end{array}\right)=t_{10}$.

Then $V_{g}\left(x^{t_{1}}\right)=x^{\pi(k+m)+t^{\prime}}$ for $t^{\prime}=\arg \left(r\left(t_{0}\right) u\left(\begin{array}{l}a \\ b\end{array}\right)\right)$.

Proof. By definition we have that $V_{g}\left(x^{t_{1}}\right)=v\left(g x^{t_{1}}\right)$. Further, $g x^{t_{1}}=x^{t} u x^{t_{1}}=$ $x^{\pi(k+m)} x^{t_{0}} u x^{t_{10}}$ since $x^{\pi}$ is in the center of $\mathbb{G}$ by Theorem $2.5(\mathrm{c})$.

By Lemma 2.4 we have $u x^{t_{10}}=x^{\tilde{t}} \widetilde{u}$ with $\widetilde{u} \in \mathbb{U}$ and $\widetilde{t}=\arg \left(u r\left(t_{10}\right)\left(\begin{array}{l}1 \\ 0\end{array}\right)\right) \in[0, \pi)$. Hence, $x^{t_{0}} u x^{t_{10}}=x^{t_{0}+\widetilde{t}} \widetilde{u}$. On the other hand, $t^{\prime}=\arg \left(r\left(t_{0}\right) u\left(\begin{array}{l}a \\ b\end{array}\right)\right)=t_{0}+\widetilde{t}$, since both $t_{0}, \widetilde{t} \in[0, \pi)$. It follows that $g x^{t_{1}}=x^{\pi(k+m)+t_{0}+\tilde{t}} \widetilde{u}$ and $V_{g}\left(x^{t_{1}}\right)=$ $x^{\pi(k+m)+t^{\prime}}$.

\section{Exceptional Cones in the Universal Covering Group $\mathbb{G}$}

In this section we construct exceptional cones of type $\left(C_{k}\right)$ for every $k$ in the universal covering group $\mathbb{G}$ of $\mathrm{SL}(2, \mathbb{R})$.

We define first two particular elements $w_{1}, w_{2}$ in $\mathbb{G}$ which will play an important role in this construction. The element $w_{1}=\left(\begin{array}{ll}1 & 2 \\ 0 & 1\end{array}\right) \in \mathbb{U} \subset \mathbb{G}$ and $\tau\left(w_{1}\right)=w_{1}$ follows. Next we consider the element $\left(\begin{array}{ll}1 & 0 \\ 2 & 1\end{array}\right) \in \mathrm{SL}(2, \mathbb{R})$ and $\alpha=\arg \left[\left(\begin{array}{ll}1 & 0 \\ 2 & 1\end{array}\right)\left(\begin{array}{l}1 \\ 0\end{array}\right)\right]=\arctan 2 \in$ $(0, \pi)$ and define $w_{2}$ as $x^{\alpha} u$ where $u=r(-\alpha)\left(\begin{array}{cc}1 & 0 \\ 2 & 1\end{array}\right) \in \mathbb{U}$; hence, $\tau\left(w_{2}\right)=\left(\begin{array}{ll}1 & 0 \\ 2 & 1\end{array}\right)$.

Lemma 3.1. Let $b$ be an element in $[0, \pi)$. Then $\lim _{n \rightarrow \infty} V_{w_{1}^{n}}\left(x^{b}\right)=x^{0}$.

Proof. We consider the real number $b_{n}$ with $x^{b_{n}}=V_{w_{1}^{n}}\left(x^{b}\right)$. Since $w_{1}^{n}=\left(\begin{array}{cc}1 & 2 n \\ 0 & 1\end{array}\right)$ and $\tau\left(w_{1}^{n}\right)=w_{1}^{n}$, we can apply Lemma 2.7 and obtain

$$
b_{n}=\arg \left[\left(\begin{array}{cc}
1 & 2 n \\
0 & 1
\end{array}\right)\left(\begin{array}{cc}
\cos b \\
\sin b
\end{array}\right)\right]=\arg \left(\begin{array}{c}
\cos b+2 n \sin b \\
\sin b
\end{array}\right) .
$$

If $b=0$, then $b_{n}=0$ for all $n \geq 0$ and the result follows. If $b \in(0, \pi)$, then $\sin b>0$ and $\lim _{n \rightarrow \infty}(\cos b+2 n \sin b)=\infty$; the statement of the lemma follows.

We are now ready to define one of the main objects of this paper:

$$
\mathbb{P}=\left\{g \in \mathbb{G} \mid V_{g}\left(x^{0}\right) \geq x^{0}\right\} .
$$

The next result shows that this is an exceptional cone of type $\left(\mathrm{C}_{1}\right)$ in $\mathbb{G}$.

Theorem 3.2. a): The set $\mathbb{P}=\left\{g \in \mathbb{G} \mid V_{g}\left(x^{0}\right) \geq x^{0}\right\}$ is a cone in $\mathbb{G}$ with $U(\mathbb{P})=$ $\mathbb{U}$.

b): Any right $\mathbb{P}$-ideal is either a principal right ideal $x^{t} \mathbb{P}$ or of the form $x^{t} J(\mathbb{P})$ for some $t \in \mathbb{R}$.

c): Any $\mathbb{P}$-ideal has the form $x^{\pi m} \mathbb{P}$ or $x^{\pi m} J(\mathbb{P})$ for some $m$ in $\mathbb{Z}$.

d): The cone $\mathbb{P}$ is exceptional of rank one with $Q=x^{\pi} \mathbb{P}$ the prime ideal that is not completely prime; $\mathbb{P}$ is exceptional of type $\left(\mathrm{C}_{1}\right)$. 
Proof. a) If $g$ and $h$ are elements in $\mathbb{P}$, then $V_{g h}\left(x^{0}\right)=V_{g}\left(V_{h}\left(x^{0}\right)\right) \geq V_{g}\left(x^{0}\right) \geq x^{0}$ by Lemma 2.6 , a) and d), and $g h \in \mathbb{P}$ follows.

If $g$ is not in $\mathbb{P}$, then $V_{g}\left(x^{0}\right)<x^{0}$; hence, $x^{0}<V_{g^{-1}}\left(x^{0}\right)$ again by Lemma 2.6, and $g^{-1} \in \mathbb{P}$ follows and $\mathbb{P}$ is a cone of $\mathbb{G}$. It also follows from the above arguments that $g, g^{-1} \in \mathbb{P}$ implies $V_{g}\left(x^{0}\right)=x^{0}$ and $g \in \mathbb{U}$. Conversely, $\mathbb{U} \subset \mathbb{P}$ and $U(\mathbb{P})=\mathbb{U}$ follows. Hence, $J(\mathbb{P})=\left\{g \in \mathbb{G} \mid V_{g}\left(x^{0}\right)>x^{0}\right\}$.

b) Let $I$ be any right $\mathbb{P}$-ideal in $\mathbb{G}$. Then it follows that $x^{\alpha}=\inf \left\{V_{g}\left(x^{0}\right) \mid g \in I\right\}$ exists since $I \subseteq c \mathbb{P}$ for some $c \in \mathbb{G}$. We will show that $\widehat{I}=x^{\alpha} \mathbb{P}$ for the divisorial closure $\widehat{I}$ of $I$, see Definition 1.4. By definition we have $x^{\alpha} \mathbb{P} \supseteq g \mathbb{P}=x^{\beta} \mathbb{P}$ for all $g \in I$ since $\alpha \leq \beta$; hence $x^{\alpha} \mathbb{P} \supseteq I$. Conversely, if $h \in \mathbb{G}$ with $h \mathbb{P}=x^{\gamma} \mathbb{P} \supseteq I$, then $\gamma \leq V_{g}\left(x^{0}\right)$ for all $g$ in $I$ and $\gamma \leq \alpha$ follows; hence $\widehat{I}=x^{\alpha} \mathbb{P}$. It follows that either $I=x^{\alpha} \mathbb{P}=\widehat{I}$ or that $\widehat{I}=x^{\alpha} \mathbb{P}$ and $I=x^{\alpha} J(P)$; see Property d) in Section 1.2.

c) Assume that $x^{t} \mathbb{P}$ is a $\mathbb{P}$-ideal. For $t=\pi m+t_{0}, m \in \mathbb{Z}$ and $t_{0} \in[0, \pi)$ it follows that $x^{t_{0}} \mathbb{P}$ is also a $\mathbb{P}$-ideal since $x^{\pi}$ is central in $\mathbb{G}$. If $t_{0}>0$, it follows from Lemma 3.1 that there exists a power $w_{1}^{n}$ of $w_{1}$ in $\mathbb{U}$ with $w_{1}^{n} x^{t_{0}} \mathbb{P} \supset x^{t_{0}} \mathbb{P}$, a contradiction that shows that $x^{t} \mathbb{P}=x^{\pi m} \mathbb{P}$. If $I=x^{t} J(\mathbb{P})$ is a $\mathbb{P}$-ideal, then $\widehat{I}=x^{t} \mathbb{P}$ is a $\mathbb{P}$-ideal by Property d) in Section 1.2 , and $t=\pi m$ by the above argument.

d) We have $\mathbb{P} \supset J(\mathbb{P}) \supset x^{\pi} \mathbb{P}=Q$ and $Q$ is not a completely prime ideal of $\mathbb{P}$, since $x^{\pi / 2} \cdot x^{\pi / 2} \in Q$, but $x^{\pi / 2} \notin Q$. However, $Q$ is a prime ideal, since any ideals $A$ and $B$ of $\mathbb{P}$ that contain $Q$ properly, also contain $J(\mathbb{P})$; hence, $A B \supseteq J(\mathbb{P}) J(\mathbb{P})=$ $J(\mathbb{P}) \supset Q$, and it follows that $Q$ is a prime ideal that is not completely prime. There are no further ideals between $J(\mathbb{P})$ and $Q$, and $\bigcap Q^{n}=\emptyset$. It follows that $\mathbb{P}$ is an exceptional cone of type $\left(\mathrm{C}_{1}\right)$; see Theorem 1.9.

We denote by $F$ the subgroup gr $\left\{w_{1}, w_{2}\right\}$ of $\mathbb{G}$ generated by $w_{1}$ and $w_{2}$. This subgroup is mapped by $\tau$ onto the subgroup gr $\left\{\left(\begin{array}{ll}1 & 2 \\ 0 & 1\end{array}\right),\left(\begin{array}{ll}1 & 0 \\ 2 & 1\end{array}\right)\right\}$ of $\mathrm{SL}(2, \mathbb{R})$ generated by $\left(\begin{array}{ll}1 & 2 \\ 0 & 1\end{array}\right)$ and $\left(\begin{array}{ll}1 & 0 \\ 2 & 1\end{array}\right)$. Since this subgroup of $\operatorname{SL}(2, \mathbb{R})$ is free (see [15], 14.2.1), the group $F$ is free of rank 2 .

Lemma 3.2. Let $h_{1}$ be the element $w_{2} w_{1}^{-1} w_{2}$ in F. Then $V_{h_{1}}\left(x^{0}\right)=x^{\arg \left(\begin{array}{l}3 \\ 4\end{array}\right)+\pi} \in$ $\left(x^{\pi}, x^{\pi+\frac{\pi}{2}}\right)$.

Proof. We have $w_{2} x^{0}=x^{\alpha} u$ for $\alpha=\arg \left(\begin{array}{l}1 \\ 2\end{array}\right) \in(0, \pi)$ with

$$
u=r(-\alpha)\left(\begin{array}{ll}
1 & 0 \\
2 & 1
\end{array}\right) \in \mathbb{U} \text {. }
$$

It follows from Lemma 2.7 that

$$
V_{w_{1}^{-1}}\left(x^{\alpha}\right)=x^{t^{\prime}}=x^{\arg \left(\begin{array}{c}
-3 \\
2
\end{array}\right)}, \text { since } w_{1}^{-1}=\left(\begin{array}{cc}
1 & -2 \\
0 & 1
\end{array}\right) \in \mathbb{U}
$$

and

$$
t^{\prime}=\arg \left[\left(\begin{array}{cc}
1 & -2 \\
0 & 1
\end{array}\right)\left(\begin{array}{l}
1 \\
2
\end{array}\right)\right]=\arg \left(\begin{array}{c}
-3 \\
2
\end{array}\right) .
$$

By a further application of Lemma 2.7 we obtain

$$
V_{h_{1}}\left(x^{0}\right)=V_{w_{2}}\left(x^{\arg \left(\begin{array}{c}
-3 \\
2
\end{array}\right)}\right)=x^{t^{\prime \prime}}
$$

with

$$
t^{\prime \prime}=\arg \left[\tau\left(w_{2}\right)\left(\begin{array}{c}
-3 \\
2
\end{array}\right)\right]=\arg \left[\left(\begin{array}{cc}
1 & 0 \\
2 & 1
\end{array}\right)\left(\begin{array}{c}
-3 \\
2
\end{array}\right)\right]=\arg \left(\begin{array}{l}
-3 \\
-4
\end{array}\right) .
$$

Hence, $V_{h_{1}}\left(x^{0}\right)=x^{\arg \left(\begin{array}{c}-3 \\ -4\end{array}\right)}=x^{\pi+\arg \left({ }_{4}^{3}\right)}$, which proves the lemma. 
In order to construct further cones we consider a subgroup $H$ of $\mathbb{G}$ that contains $F$ and define

$$
P_{H}=H \cap \mathbb{P}
$$

It follows immediately that $P_{H}$ is closed under multiplication. If $g \in H \backslash P_{H}$, then $g \notin \mathbb{P}$ and $g^{-1} \in H \cap \mathbb{P}=P_{H}$ follows; $P_{H}$ is a cone of $H$.

Lemma 3.3. Let $t \in \mathbb{R}$. Then $P_{H} x^{t} \mathbb{P}=\mathbb{P} x^{t} \mathbb{P}$.

Proof. It is enough to prove this for $t \in[0, \pi)$, since $t=k \pi+t_{0}, t_{0} \in[0, \pi)$ in the general case with $x^{\pi}$ in the center of $G$.

If $t=0$, then $P_{H} x^{t} \mathbb{P}=\mathbb{P}=\mathbb{P} x^{t} \mathbb{P}$. If $t \in(0, \pi)$, then for any $j \in J(\mathbb{P})$ there exists an $n$ with $w_{1}^{n} x^{t} \mathbb{P} \supseteq j \mathbb{P}$ by Lemma 3.1. Hence, $\mathbb{P} x^{t} \mathbb{P}=J(\mathbb{P})=\bigcup w_{1}^{n} x^{t} \mathbb{P} \subseteq P_{H} x^{t} \mathbb{P}$, and the statement in the lemma follows.

The next result shows that $F$ contains elements of a certain type.

Lemma 3.4. For any integer $m$ and any $\varepsilon>0$ there exists an element $x^{t} u$ in $F$ with $u \in \mathbb{U}$ and $t \in(\pi m, \pi m+\varepsilon)$.

Proof. Let $h_{1}$ be the element $w_{2} w_{1}^{-1} w_{2}$ in $F$. Then, by Lemma 3.3, we have $V_{h_{1}^{-1}}\left(x^{\beta+\pi}\right)=x^{0}$ where $\beta=\arg \left(\begin{array}{l}3 \\ 4\end{array}\right)$. It follows that $V_{h_{1}^{-1}}\left(x^{0}\right)<V_{h_{1}^{-1}}\left(x^{\beta}\right)=x^{-\pi}$ and that $V_{h_{1}^{-N}}\left(x^{0}\right)<x^{-\pi N}$ for any natural number $N$.

We conclude that for the given integer $m$ there exists a natural number $N$ and an integer $M<m$ with

$$
V_{h_{1}^{-N}}\left(x^{0}\right) \in\left[x^{\pi M}, x^{\pi(M+1)}\right) .
$$

For $\varepsilon$ the given real number, there exists by Lemma 3.3 and the continuity of $V_{g}$ a $\delta$ with $0<\delta<\varepsilon$ and

and hence

$$
V_{h_{1}}\left(\left[x^{0}, x^{\delta}\right)\right) \subseteq\left(x^{\pi}, x^{\pi+\frac{\pi}{2}}\right)
$$

$$
V_{h_{1}}\left(\left[x^{\pi k}, x^{\pi k+\delta}\right)\right) \subseteq\left(x^{\pi(k+1)}, x^{\pi(k+1)+\frac{\pi}{2}}\right)
$$

follows for all $k \in \mathbb{Z}$.

By Lemma 3.1 there exists a natural number $n_{1}$ with

$$
V_{w_{1}^{n_{1}} h}\left(x^{0}\right) \in\left[x^{\pi M}, x^{\pi M+\delta}\right) \quad \text { and } \quad h=h_{1}^{-N} \in F .
$$

Hence, by $(*)$ we obtain

$$
V_{h_{1} w_{1}^{n_{1}} h}\left(x^{0}\right) \in\left(x^{\pi(M+1)}, x^{\pi(M+1)+\frac{\pi}{2}}\right) .
$$

By another application of Lemma 3.1, there exists a natural number $n_{2}$ with

$$
V_{w_{1}^{n_{2}} h_{1} w_{1}^{n_{1}} h}\left(x^{0}\right) \in\left(x^{\pi(M+1)}, x^{\pi(M+1)+\delta}\right) \subseteq\left(x^{\pi(M+1)}, x^{\pi(M+1)+\varepsilon}\right) .
$$

By repeating the last two steps $m-(M+1)$ times, the statement of the lemma follows.

The next result shows that the cones $P_{H}$ are indeed exceptional.

Proposition 3.5. Let $H \supseteq F$ be a subgroup of $\mathbb{G}$ and $P_{H}=\mathbb{P} \cap H$. Then:

a): $P_{H}$ is an exceptional rank one cone in $H$.

b): The mapping $\varphi: \mathcal{M}(\mathbb{P}) \rightarrow \mathcal{M}\left(P_{H}\right)$ with $\varphi\left(x^{\pi m} \mathbb{P}\right)=x^{\pi m} \mathbb{P} \cap H, m \in \mathbb{Z}$, defines an isomorphism between $\mathcal{M}(\mathbb{P})$ and $\mathcal{M}\left(P_{H}\right)$. The inverse of $\varphi$ is given by $\varphi^{-1}(C)=\widehat{C P}$ for $C$ a divisorial $P_{H}$-ideal. 
Proof. We recall that $\mathcal{M}(\mathbb{P})$ is the group of divisorial $\mathbb{P}$-ideals in $\mathbb{G}$ (Definition 1.6) and that $\mathcal{M}(\mathbb{P})=\operatorname{gr}\{Q\}=\operatorname{gr}\left(x^{\pi} \mathbb{P}\right)$ by Theorems 1.9 and 3.2.

If $C$ is a divisorial $P_{H}$-ideal in $H$, then $C \mathbb{P}$ is a $\mathbb{P}$-ideal in $\mathbb{G}$ by Lemma 3.4. The divisorial closure $\widehat{C P}$ of $C \mathbb{P}$ is therefore equal to some power of $x^{\pi} \mathbb{P}$ and $\widehat{C P}=x^{\pi m} \mathbb{P}$ follows for some $m$ in $\mathbb{Z}$. We want to prove that $\widehat{C P} \cap H=C$ and assume that $h P_{H} \supseteq C$ for some $h \in H$. Then $h P_{H} \mathbb{P}=h \mathbb{P} \supseteq C \mathbb{P}$; hence $h \mathbb{P} \supseteq \widehat{C \mathbb{P}}$. Therefore, $h P_{H}=h \mathbb{P} \cap H \supseteq \widehat{C P} \cap H$. It follows that $C=\widehat{\widehat{C}} \supseteq \widehat{C P} \cap H \supseteq C$ and $C=\widehat{C P} \cap H$. This shows that $C$ being a divisorial $P_{H}$-ideal implies $C=x^{\pi m} \mathbb{P} \cap H$ for some $m$. We want to show next that $\left(x^{\pi n \mathbb{P} \cap} H\right)=x^{\pi n} \mathbb{P} \cap H$ for any $n$. Since $\left(x^{\pi n \mathbb{P} \cap} H\right)$ is divisorial, we know that $\left(x^{\pi n \mathbb{P} \cap} H\right)=x^{\pi m} \mathbb{P} \cap H$ for some $m$ by the above argument.

By Lemma 3.5 there exist elements $x^{t_{1}} u_{1}, x^{t_{2}} u_{2} \in F \subseteq H$ with $t_{1}<t_{2} \in$ $\mathbb{R}, u_{1}, u_{2} \in \mathbb{U}$ and $t_{1}, t_{2} \in\left(\pi(n-1), \pi(n-1)+\frac{\pi}{2}\right)$.

It follows that

$$
x^{t_{1}} u_{1} P_{H}=x^{t_{1}} u_{1} \mathbb{P} \cap H \supseteq x^{t_{2}} u_{2} \mathbb{P} \cap H=x^{t_{2}} u_{2} P_{H} \supseteq x^{\pi n} \mathbb{P} \cap H .
$$

Hence, $x^{\pi(n-1)} \mathbb{P} \cap H \supseteq\left(x^{\pi \hat{n} \mathbb{P} \cap} H\right) \supseteq x^{\pi n} \mathbb{P} \cap H$.

If $x^{\pi(n-1)} \mathbb{P} \cap H=\left(x^{\pi n \mathbb{P} \cap} H\right)$, then this ideal would also be equal to $x^{t_{1}} u_{1} P_{H}$ and $x^{t_{2}} u_{2} P_{H}$. This would imply $x^{t_{1}} u_{1} P_{H} \mathbb{P}=x^{t_{1}} \mathbb{P}=x^{t_{2}} u_{2} P_{H} \mathbb{P}=x^{t_{2}} \mathbb{P}$, a contradiction that shows that $\left(x^{\pi n \mathbb{P} \cap} H\right)=\left(x^{\pi n} \mathbb{P} \cap H\right)$ for all $n$. This set of divisorial $P_{H}$-ideals does not contain $J\left(P_{H}\right)$, does not contain a completely prime ideal (Lemmas 3.3 and 3.5) and no ideal of the form $a J\left(P_{H}\right) \neq J\left(P_{H}\right), a \in P_{H}$, is completely prime in $P_{H}$. This shows that $P_{H}$ has rank one and that $\mathcal{M}\left(P_{H}\right)$ is infinite cyclic with $Q_{H}=x^{\pi} \mathbb{P} \cap H$ as the positive generator of $\mathcal{M}\left(P_{H}\right)$. Since $J\left(P_{H}\right) \supset Q_{H}$, it follows from Theorem 1.9 that $P_{H}$ is an exceptional rank one cone in $H$. This proves all statements in the lemma. $\left(C_{k}\right)$.

We consider now a condition that will guarantee that $P_{H}$ is exceptional of type

Proposition 3.6. Let $H$ be a subgroup of $\mathbb{G}$ containing $F$ with $H \cap\left(\operatorname{gr}\left\{x^{\pi}\right\} \times \mathbb{U}\right)=$ $\operatorname{gr}\left\{x^{\pi k}\right\} \times U\left(P_{H}\right)$ for some integer $k \geq 0$. Then the exceptional cone $P_{H}$ has type $\left(C_{k}\right)$.

Proof. It was shown in the previous proposition that $P_{H}$ is an exceptional cone with $\mathcal{M}\left(P_{H}\right)=\operatorname{gr}\left\{\left(x^{\pi} \mathbb{P} \cap H\right)\right\}$. To prove the statement in this proposition it must be shown that $\mathcal{H}\left(P_{H}\right)=\operatorname{gr}\left\{x^{\pi k} P_{H}\right\}$, see Theorem 1.9. Hence, let $g P_{H}=P_{H} g$ be a principal ideal in $H$ (see property e) in Section 1.1).

Then $g \mathbb{P}=P_{H} g \mathbb{P}=\mathbb{P} g \mathbb{P}$ by Lemma 3.4 and $g \mathbb{P}=\mathbb{P} g$ since $\mathbb{P}$ has rank one. By Theorem 3.2, c) it follows that $g=x^{\pi m} u \in H$ for some integer $m$ and $u \in \mathbb{U}$ and $g=x^{\pi k n} u$ for $u \in U\left(P_{H}\right)$ and some integer $n$ by assumption. Therefore, $g P_{H}=x^{\pi k n} P_{H}$ and $\mathcal{H}\left(P_{H}\right)=\operatorname{gr}\left\{x^{\pi k} P_{H}\right\}=\operatorname{gr}\left\{\widehat{Q^{k}}\right\}$ follows for $Q=x^{\pi} \mathbb{P} \cap H ; P_{H}$ is exceptional of type $\left(C_{k}\right)$.

Theorem 3.7. Let $H_{k}=\operatorname{gr}\left\{w_{1}, w_{2}, x^{\pi k}\right\}$ be the subgroup of $\mathbb{G}$ generated by $F$ and the central element $x^{\pi k}$ for an integer $k \geq 0$. Then $P_{k}=\mathbb{P} \cap H_{k}$ is an exceptional rank one cone in $H_{k}$ of type $\left(C_{k}\right)$.

Proof. It is sufficient to verify the conditions in Proposition 3.7 for $H_{k}$. 
Assume that

$$
x^{\pi k p} w_{1}^{\nu_{1}} w_{2}^{\mu_{1}} w_{1}^{\nu_{2}} w_{2}^{\mu_{2}} \cdots w_{1}^{\nu_{n}} w_{2}^{\mu_{n}}=x^{\pi m} u \in H_{k} \cap\left(\operatorname{gr}\left\{x^{\pi}\right\} \times \mathbb{U}\right)
$$

for some integers $p, \nu_{i}, \mu_{i}$ for $i=1, \ldots, n$, and $u \in \mathbb{U}$. We apply the mapping $\tau$ (Theorem 2.5b)) to both sides of the above equation and obtain

$$
(-1)^{k p}\left(\begin{array}{cc}
1 & 2 \nu_{1} \\
0 & 1
\end{array}\right)\left(\begin{array}{cc}
1 & 0 \\
2 \mu_{1} & 1
\end{array}\right) \cdots\left(\begin{array}{cc}
1 & 2 \nu_{n} \\
0 & 1
\end{array}\right)\left(\begin{array}{cc}
1 & 0 \\
2 \mu_{n} & 1
\end{array}\right)=(-1)^{m}\left(\begin{array}{cc}
a & b \\
0 & a^{-1}
\end{array}\right)
$$

where $u=\left(\begin{array}{cc}a & b \\ 0 & a^{-1}\end{array}\right)$ with $b, 0<a \in \mathbb{R}$.

Since the entries of the matrices at the left side are all integers, it follows that $a$ and $a^{-1}$ are integers greater than zero; hence $a=a^{-1}=1$. By a similar argument it follows that $b$ is an even integer, $b=2 s$ for some $s$ in $\mathbb{Z}$ and $u=\left(\begin{array}{ll}1 & 2 s \\ 0 & 1\end{array}\right)=\left(\begin{array}{ll}1 & 2 \\ 0 & 1\end{array}\right)^{s}=$ $w_{1}^{s} \in \tau(F)$ follows.

If $(-1)^{k p}(-1)^{m}=-1$, then it follows from $(* *)$ that

$$
-\left(\begin{array}{ll}
1 & 0 \\
0 & 1
\end{array}\right)=\left(\begin{array}{cc}
1 & 2 \nu_{1} \\
0 & 1
\end{array}\right)\left(\begin{array}{cc}
1 & 0 \\
2 \mu_{1} & 1
\end{array}\right) \cdots\left(\begin{array}{cc}
1 & 2 \nu_{n} \\
0 & 1
\end{array}\right)\left(\begin{array}{cc}
1 & 0 \\
2 \mu_{n} & 1
\end{array}\right)\left(\begin{array}{cc}
1 & -b \\
0 & 1
\end{array}\right) \in \tau(F),
$$

which is a contradiction, since the group $\tau(F)$ freely generated by $\tau\left(w_{1}\right)$ and $\tau\left(w_{2}\right)$ (see the remarks before Lemma 3.3) does not contain a nontrivial central element.

Therefore, $(-1)^{k p}=(-1)^{m}$ can be cancelled in $(* *)$ and, using again the fact that $\tau\left(w_{1}\right)=\left(\begin{array}{ll}1 & 2 \\ 0 & 1\end{array}\right)$ and $\tau\left(w_{2}\right)=\left(\begin{array}{ll}1 & 0 \\ 2 & 1\end{array}\right)$ are free generators of $\tau(F)$, it follows that $n=1$, $b=2 \nu_{1}$ if we ignore exponents that could be zero. With $u=w_{1}^{\nu_{1}}$ we can rewrite (*) as: $x^{\pi k p} w_{1}^{\nu_{1}}=x^{\pi m} w_{1}^{\nu_{1}}$. It follows that $\nu_{1}=s, m=k p$ and $u=w_{1}^{s} \in U\left(P_{k}\right)$; the condition in Proposition 3.7 is satisfied and Theorem 3.8 follows.

\section{Examples of Exceptional Rank One Chain Domains}

In this section we construct domains $S_{k}$ associated with the exceptional cones $P_{k}$ of type $\left(C_{k}\right)$ as described in Theorem 3.8.

In Lemma 2.6(c) it was proved that $T \mathbb{U}$ is an Ore domain for any skew field $T$ and the subgroup $\mathbb{U}$ of $\mathbb{G}$. We denote by $K$ the skew field of quotients of $T \mathbb{U}$ for a given skew field $T$; for example, $T=\mathbb{Q}$, the rationals. Let $K\{\mathbb{G}\}$ be the right $K$-vector space and left $T$-vector space consisting of all series

$$
\gamma=x^{t_{1}} k_{1}+x^{t_{2}} k_{2}+\cdots
$$

with $t_{1}<t_{2}<\ldots, k_{i} \in K$, and $\operatorname{supp}(\gamma)=\left\{x^{t_{i}} \mid k_{i} \neq 0\right\}$ well ordered.

We call $\operatorname{supp}(\gamma)$ the support of the series $\gamma$. If $k_{1} \neq 0$, then $v(\gamma)=x^{t_{1}} \in R$ is the norm of $\gamma$ and $v(0)=\infty$ for $\gamma=0$.

Let $Q=$ End $K\{\mathbb{G}\}_{K}$ be the endomorphism ring of the $K$-vector space $K\{G\}_{K}$. For $q \in Q$ and $\gamma \in K\{\mathbb{G}\}$ we write $q[\gamma]$ for the image of $\gamma$ under $q$. The representation $V: \mathbb{G} \rightarrow \operatorname{Aut}(R, \geq)$ considered in Section 2.3 can be extended to a mapping $V$ defined on $Q$ with

$$
V_{q}\left(x^{t}\right)=v\left(q\left[x^{t}\right]\right), \quad V_{q}(\infty)=\infty
$$

for $q \in Q, x^{t} \in R$, and $V_{q}:(R, \infty) \rightarrow(R, \infty)$. It follows that

$$
V_{a+b}\left(x^{t}\right) \geq \min \left\{V_{a}\left(x^{t}\right), V_{b}\left(x^{t}\right)\right\}
$$

for any $a, b \in Q$ and $x^{t} \in R$. However, $V_{a b}$ is not equal to $V_{a} \circ V_{b}$ in general.

We recall a definition and a result given by Mathiak in [17].

Definition 4.1. Let $D$ be a skew field and $(\Gamma, \leq)$ a linearly ordered set. Then a mapping $V: D^{*} \rightarrow$ Aut $(\Gamma, \leq)$ is called an $M$-valuation if the following conditions hold:

MV1. $V_{a b}=V_{a} \circ V_{b}$ for any $a, b \in D^{*}$; 
MV2. $V_{a+b}(h) \geq \min \left\{V_{a}(h), V_{b}(h)\right\}$ for any $a, b \in D^{*}$ with $a+b \neq 0$ and $h \in \Gamma$.

If we add the symbol $\infty$ for infinity to $\Gamma$ and define $V_{0}(h)=\infty$ and $V_{a}(\infty)=\infty$ for all $h \in \Gamma, 0, a \in D$, then MV1 and MV2 will be valid for all elements $a, b \in D$ and all $h \in \Gamma \cup\{\infty\}$.

The next result follows almost directly from the previous definition; see also [16 and $[17$.

Proposition 4.2. Let $V: D^{*} \rightarrow \operatorname{Aut}(\Gamma, \leq)$ be an $M$-valuation for a skew field $D$ and a linearly ordered set $(\Gamma, \leq)$ and let $h$ be an element in $\Gamma$.

Then the set $S_{h}=\left\{d \in D \mid V_{d}(h) \geq h\right\}$ is a total subring of D. Conversely, any total subring $S$ in a skew field $D$ can be obtained in this way for $\Gamma=\left\{a S \mid a \in D^{*}\right\}$, $a S \geq b S$ if and only if $a S \subseteq b S$ and $V_{d}(a S)=d a S$. The ring $S$ coincides with $S_{h}$ for $h=S \in \Gamma$.

The space $K\{\mathbb{G}\}$ introduced above is also a left $\mathbb{G}$-module if we define for $g \in \mathbb{G}$ and $\gamma=\sum x^{t_{1}} k_{i} \in K\{\mathbb{G}\}$ that

$$
g \gamma=x^{t_{1}^{\prime}}\left(u_{1} k_{1}\right)+x^{t_{2}^{\prime}}\left(u_{2} k_{2}\right)+x^{t_{3}^{\prime}}\left(u_{3} k_{3}\right)+\ldots
$$

where $g \cdot x^{t_{i}}=x^{t_{i}^{\prime}} u_{i}$ for $u_{i} \in \mathbb{U} \subseteq K, t_{i}^{\prime} \in \mathbb{R}$. It follows from Lemma 2.6(d) that $t_{1}^{\prime}<t_{2}^{\prime}<t_{3}^{\prime}<\ldots$ is also well ordered and hence $g \gamma \in K\{\mathbb{G}\}$. The group ring $T \mathbb{G}$ can therefore be considered as a subring of $Q$.

If $A$ is any subring of $Q$, then we define $\mathcal{D}[0, A]=A$ and $\mathcal{D}[n+1, A]$ as the subring of $Q$ generated by $\mathcal{D}[n, A]$ and all inverses of elements of $\mathcal{D}[n, A]$ in $Q$. The union

$$
\bigcup_{n=0}^{\infty} \mathcal{D}[n, A]=\mathcal{D}[A]
$$

is called the rational closure of $A$ in $Q$. Let $\mathbb{D}=\mathcal{D}[T \mathbb{G}]$ be the rational closure of the group ring $T \mathbb{G}$ in $Q$.

The following result can be found in 11] (see [10 also):

Theorem 4.3. a) The rational closure $\mathbb{D}$ of $T \mathbb{G}$ in $Q$ is a skew field.

b) The mapping $V$ restricted to $\mathbb{D}^{*}$ is an $M$-valuation of $\mathbb{D}^{*}$ to Aut $(R, \leq)$.

c) The ring $S=\left\{d \in \mathbb{D} \mid V_{d}\left(x^{0}\right) \geq x^{0}\right\}$ is an exceptional rank one chain order in $\mathbb{D}$ of type $\left(C_{1}\right)$ associated with the exceptional cone $\mathbb{P}$ in the group $\mathbb{G}$.

In order to construct skew fields that contain rank one exceptional chain orders of type $\left(C_{k}\right)$ we consider the rational closure $D_{k}=\mathcal{D}\left[T H_{k}\right]$ of the group ring $T H_{k}$ for the group $H_{k}=\operatorname{gr}\left\{w_{1}, w_{2}, x^{\pi k}\right\}$ (see Theorem 3.8) in $Q=$ End $K\{\mathbb{G}\}_{K}$.

Since $D_{k} \subseteq \mathbb{D}=\mathcal{D}[T \mathbb{G}] \subset Q$ and $\mathbb{D}$ is a skew field by the above theorem, it follows that $D_{k}$ is also a skew field and $S_{k}=S \cap D_{k}$ is a total subring of $D_{k}$.

It follows from Corollary 1.10 and Theorem 3.8 that $S_{k}$ is an exceptional rank one chain domain of type $\left(C_{k}\right)$ if the following theorem is proved:

Theorem 4.4. The total subring $S_{k}=S \cap D_{k}$ is associated with the cone $P_{k}=$ $\mathbb{P} \cap H_{k}$.

Before this theorem can be proved, we need the result in the following lemma.

Lemma 4.5. Let $\gamma \in K\{\mathbb{G}\}$. Then

$$
\bigcup_{d \in D_{k}} \operatorname{supp} d[\gamma] \subseteq \bigcup_{g \in H_{k}} V_{g}(\operatorname{supp} \gamma)
$$


Proof. Let $Y_{\gamma}$ be the right side in $(*)$. Then in order to prove $(*)$ it is sufficient to prove

$$
\operatorname{supp} d[\gamma] \subseteq Y_{\gamma}
$$

for any $\gamma \in K\{\mathbb{G}\}$ and any $d \in D_{k}=\bigcup \mathcal{D}\left[n, T H_{k}\right]$. We will prove this in five steps using induction on $n$ for $n$ the smallest index with $d \in \mathcal{D}\left[n, T H_{k}\right]$.

STEP 1. Assume that $d=x^{t} u \in H_{k}, u \in \mathbb{U}$ and that $\gamma=\sum_{i<\Lambda} x^{t_{i}} k_{i} \in K\{\mathbb{G}\}$, $0 \neq k_{i} \in K$ for all ordinals $i<\Lambda$.

Then $d \gamma=\sum_{i<\Lambda} x^{t^{\prime}}\left(u_{i} k_{i}\right)$ for $x^{t} u x^{t_{i}}=x^{t_{i}^{\prime}} u_{i} \in \mathbb{G}, u_{i} \in \mathbb{U}$. Hence, supp $d[\gamma]=$ $\operatorname{supp} d \gamma=\left\{x^{t_{i}^{\prime}} \mid i<\Lambda\right\}=\left\{V_{d}\left(x^{t_{i}}\right) \mid i<\Lambda\right\} \subseteq\left\{V_{g}\left(x^{t_{i}}\right) \mid g \in H_{k}, i<\Lambda\right\}=Y_{\gamma}$.

STEP 2. The inclusion (**) follows immediately for $d \in T$.

STEP 3. Assume that $a, b \in D_{k}$ with $\operatorname{supp} a[\gamma] \cup \operatorname{supp} b[\gamma] \subseteq Y_{\gamma}$ for any $\gamma \in K\{\mathbb{G}\}$. Then $\operatorname{supp}(a+b)[\gamma] \subseteq \operatorname{supp} a[\gamma] \cup \operatorname{supp} b[\gamma] \subseteq Y_{\gamma}$. Further, $V_{g}\left(Y_{\gamma}\right)=Y_{\gamma}$ for $g \in H_{k}$ and hence

$$
\operatorname{supp}(a \cdot b)[\gamma] \subseteq Y_{b[\gamma]}=\bigcup_{g \in H_{k}} V_{g}(\operatorname{supp} b[\gamma]) \subseteq \bigcup_{g \in H_{k}} V_{g}\left(Y_{\gamma}\right)=Y_{\gamma} .
$$

STEP 4. It follows from Steps 1-3 that the statement (**) is true for all $d \in T H_{k}=$ $\mathcal{D}\left[0, T H_{k}\right]$ and any $\gamma \in K\{\mathbb{G}\}$.

STEP 5. Assume that $(* *)$ is true for elements $d \in \mathcal{D}\left[n-1, T H_{k}\right]$ for some $n \geq 1$ and all $\gamma \in K\{\mathbb{G}\}$.

Let $d=p^{-1} \in \mathcal{D}\left[n, T H_{k}\right]$ with $p \in \mathcal{D}\left[n-1, T H_{k}\right]$. We consider $\beta=d[\gamma]$ and decompose $\beta$ into the sum $\beta=\beta_{0}+\beta_{1}$ with $\operatorname{supp}\left(\beta_{0}\right) \subseteq Y_{\gamma}$ and $\operatorname{supp}\left(\beta_{1}\right) \cap Y_{\gamma}=\emptyset$.

Then $\gamma=p[\beta]=p\left[\beta_{0}\right]+p\left[\beta_{1}\right]$.

By the induction hypothesis, it follows that

$$
\operatorname{supp}\left(p\left[\beta_{0}\right]\right) \subseteq \bigcup_{g \in H_{k}} V_{g}\left(\operatorname{supp} \beta_{0}\right) \subseteq \bigcup_{g \in H_{k}} V_{g}\left(Y_{\gamma}\right) \subseteq Y_{\gamma} .
$$

Hence, $\operatorname{supp}\left(p\left[\beta_{1}\right]\right)=\operatorname{supp}\left(\gamma-p\left[\beta_{0}\right]\right) \subseteq \operatorname{supp} \gamma \cup \operatorname{supp}\left(p\left[\beta_{0}\right)\right) \subseteq Y_{\gamma}$. On the other hand, $\operatorname{supp}\left(p\left[\beta_{1}\right]\right) \subseteq Y_{\beta_{1}}$ since $p \in \mathcal{D}\left[n-1, T H_{k}\right]$. If we assume that there exists an element $h$ in $\operatorname{supp}\left(p\left[\beta_{1}\right]\right)$, then, on the one hand,

$$
h=V_{g} h^{\prime} \quad \text { for some } \quad g \in H_{k} \quad \text { and some } \quad h^{\prime} \in \operatorname{supp}(\gamma)
$$

and on the other hand,

$$
h=V_{g^{\prime}}\left(h^{\prime \prime}\right) \quad \text { for some } \quad g^{\prime} \in H_{k} \quad \text { and some } \quad h^{\prime \prime} \in \operatorname{supp}\left(\beta_{1}\right) .
$$

This implies $h^{\prime \prime}=V_{\left(g^{\prime}\right)^{-1} g}\left(h^{\prime}\right) \in Y_{\gamma} \cap \operatorname{supp}\left(\beta_{1}\right)=\emptyset$, a contradiction that shows that $\operatorname{supp}\left(\beta_{1}\right)$ is empty and $\operatorname{supp}(\beta)=\operatorname{supp}\left(\beta_{0}\right) \subseteq Y_{\gamma}$.

The ring $\mathcal{D}\left[n, T H_{k}\right]$ is generated by $\mathcal{D}\left[n-1, T H_{k}\right]$ and all elements $p^{-1}$ for $p \in \mathcal{D}\left[n-1, T H_{k}\right] \backslash\{0\}$, and it now follows by an application of Step 3 that (**) is true for all elements in $\mathcal{D}\left[n, T H_{k}\right]$ which completes the induction and proves the lemma (see also: [1]).

We now return to the proof of Theorem 4.4.

Let $d$ be a nonzero element in $D_{k}$. Since $D_{k} \subseteq \mathbb{D}$ and $S$ is associated with the cone $\mathbb{P}$, the element $d$ can be decomposed as follows:

$$
d=x^{t} m=q x^{t^{\prime}} \quad \text { with } \quad m, q \in U(S), \mathbb{P} x^{t} \mathbb{P}=\mathbb{P} x^{t^{\prime}} \mathbb{P}
$$


(see Definition 1.2). It follows from $(*)$ in Lemma 4.5 with $\gamma=x^{0}$ that

$$
\operatorname{supp} d\left[x^{0}\right] \subseteq \bigcup_{g \in H_{k}} V_{g}\left(x^{0}\right)=\bigcup_{g \in H_{k}} v(g) .
$$

Hence, $v\left(d\left[x^{0}\right]\right)=V_{d}\left(x^{0}\right)=V_{x^{t}} \circ V_{m}\left(x^{0}\right)=V_{x^{t}}\left(x^{0}\right)=x^{t}$ since $m \in U(S)$, and hence $x^{t}=v(g), g=x^{t} u, u \in \mathbb{U}$ for some element $g \in H_{k}$.

It follows that $d=\left(x^{t} u\right)\left(u^{-1} m\right)$ for $x^{t} u \in H_{k}$ and $u^{-1} m=\left(x^{t} u\right)^{-1} d \in D_{k}$. Further, $u^{-1} m \in \mathbb{U} \cdot U(S) \cap D_{k}=U\left(S_{k}\right)$, since $\mathbb{U} \subseteq U(S)$ and $S \cap D_{k}=S_{k}$.

Applying the same arguments to the element $d^{-1}=x^{-t^{\prime}} q^{-1}$, we conclude that there exists an element $g^{\prime} \in H_{k}$ with $g^{\prime}=x^{-t^{\prime}} w$ for some $w \in \mathbb{U}$. Hence, we obtain a decomposition

$$
d^{-1}=\left(x^{-t^{\prime}} w\right)\left(w^{-1} q^{-1}\right) \quad \text { for } \quad w^{-1} q^{-1}=\left(g^{\prime}\right)^{-1} d^{-1} \in D_{k} \cap U(S)=U\left(S_{k}\right) .
$$

This proves the first half of condition (ii) in Definition 1.2, if we write $d=$ $(q w)\left(w^{-1} x^{t^{\prime}}\right), q w \in U\left(S_{k}\right), w^{-1} x^{t^{\prime}}=\left(g^{\prime}\right)^{-1} \in H_{k}$. It remains to prove the equality

$$
P_{k} x^{t} u P_{k}=P_{k} w^{-1} x^{t^{\prime}} P_{k} .
$$

Let $w^{-1} x^{t^{\prime}}=x^{t^{\prime \prime}} u^{\prime \prime}$ for some $u^{\prime \prime} \in \mathbb{U}$ and $t^{\prime \prime} \in \mathbb{R}$. Since $S$ is associated with $\mathbb{P}$, it follows that

$$
\mathbb{P} x^{t^{\prime}} \mathbb{P}=\mathbb{P} x^{t^{\prime \prime}} \mathbb{P} .
$$

Therefore,

$$
\begin{aligned}
P_{k} x^{t} u P_{k} & =P_{k} x^{t} u\left(\mathbb{P} \cap H_{k}\right)=P_{k} x^{t} \mathbb{P} \cap H_{k} \\
& =\mathbb{P} x^{t} \mathbb{P} \cap H_{k}=\mathbb{P} x^{t^{\prime}} \mathbb{P} \cap H_{k}=\mathbb{P} x^{t^{\prime \prime}} \mathbb{P} \cap H_{k} \\
& =P_{k} x^{t^{\prime \prime}} u^{\prime \prime} \mathbb{P} \cap H_{k}=P_{k} x^{t^{\prime \prime}} u^{\prime \prime} P_{k} \\
& =P_{k} w^{-1} x^{t^{\prime}} P_{k}
\end{aligned}
$$

where we used Lemma 3.4 for the third and sixth equality.

This completes the proof of Theorem 4.4.

Corollary 4.6. The chain domain $S_{k}$ is exceptional of rank one and of type $\left(C_{k}\right)$.

\section{REFERENCES}

1. G. M. Bergman. Right-orderable groups that are not locally indicable. Pacific J. Math. $\mathbf{1 4 7}$ (1991), 243-248. MR 92e:20030

2. H. H. Brungs, H. Marubayashi, and E. Osmanagic. A classification of prime segments in simple Artinian rings. Proc. Amer. Math. Soc. 128 (2000), 3167-3175. MR 2001b:16055

3. H. H. Brungs and M. Schröder. Prime segments of skew fields, Canad. J. Math. 47 (1995), 1148-1167. MR 97c:16021

4. H. H. Brungs and G. Törner. Chain rings and prime ideals. Arch. Math. 27 (1976), 253-260. MR 54:7537

5. H. H. Brungs and G. Törner. Extensions of chain rings. Math. Zeit. 185 (1984), 93-104. MR 85d:16012

6. H. H. Brungs and G. Törner. Ideal theory of right cones and associated rings. J. Algebra 210 (1998), 145-164. MR 99k:20113

7. P. M. Cohn, Skew Fields, Cambridge University Press, 1995. MR 97d:12003

8. N. I. Dubrovin. Chain domains. Moscow Univ. Math. Bull. Ser. 1. 37(1980), 51-54. MR 81g:16004

9. N. I. Dubrovin. An example of a chain prime ring with nilpotent elements. Mat. Sbornik 48 (1984), 437-444. MR 84f: 16012

10. N. I. Dubrovin. The rational closure of group rings of left-orderable groups. Mat. Sbornik 184(7) (1993), 3-48. MR 94g:16035 
11. N. I. Dubrovin. The rational closure of group rings of left-ordered groups. SM-DU-254, Duisburg, 1994, $96 \mathrm{pp}$.

12. T. V. Dubrovin and N. I. Dubrovin. Cones in groups. Sbornik Mathematics 187(7) 1996, 1005-1019. MR 98c:20082

13. L. Fuchs. Teilweise geordnete algebraische Strukturen, Vandenhoeck und Ruprecht, Göttingen, 1966. MR 34:4386

14. V. K. Goel and S. K. Jain. $\pi$-injective modules and rings whose cyclics are $\pi$-injective. Comm. Algebra 6 (1978), 59-73. MR 58:11016

15. M. I. Kargapolov and Ju. I. Merzljakov. Fundamentals of the Theory of Groups. SpringerVerlag, 1979. MR 80k:20002

16. K. Mathiak. Zur Bewertungstheorie nicht kommutativer Körper, J. Algebra 73, (1981), No. 2, 586-600. MR 83c:12026

17. K. Mathiak. Valuations of skew fields and projective Hjelmslev spaces. Lecture Notes in Math. 1175, Springer-Verlag, 1986. MR 87g:16002

18. B. L. Osofsky. Noncommutative rings whose cyclic modules have cyclic injective hulls. Pacific J. Math. 25 (1968), 331-340. MR 38:186

19. E. C. Posner. Left valuation rings and simple radical rings. Trans. Amer. Math. Soc. 107 (1963), 458-465. MR 27:3665

Department of Mathematical and Statistical Sciences, University of Alberta, EdMONTON T6G 2G1, CANADA

E-mail address: hbrungs@math.ualberta.ca

Department of Mathematics, Vladimir State University, Gorki Str. 87, 600026 VLADIMIR, RUSSIA

E-mail address: ndubrovin@mail.ru 